\title{
Supramolecular Peptide Nanofiber Morphology Affects Mechanotransduction of Stem Cells
}

\author{
Elif Arslan, ${ }^{\dagger}$ Meryem Hatip Koc, ${ }^{\dagger}$ Ozge Uysal, ${ }^{\dagger \dagger}$ Begum Dikecoglu, $^{\dagger}$ Ahmet E. Topal, $^{\dagger}$
}

Ruslan Garifullin, ${ }^{\dagger, \S}$ Alper D. Ozkan, ${ }^{\dagger}$ Aykutlu Dana, ${ }^{\dagger}$ Daniel Hermida-Merino, ${ }^{\perp}$ Valeria Castelletto, ${ }^{\text {II }}$

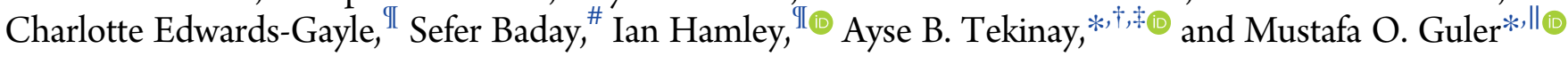

${ }^{\dagger}$ Institute of Materials Science and Nanotechnology and National Nanotechnology Research Center (UNAM) and ${ }^{\dagger}$ Neuroscience Graduate Program, Bilkent University, Ankara 06800, Turkey

${ }^{\S}$ Institute of Fundamental Medicine and Biology, Kazan Federal University, 420021 Kazan, Russian Federation

${ }^{\perp}$ ESRF - The European Synchrotron, 38000 Grenoble, France

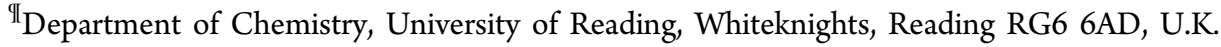

\#Applied Informatics Department, Informatics Institute, Istanbul Technical University, Istanbul 34469, Turkey

"Institute for Molecular Engineering, University of Chicago, Chicago, Illinois 60637, United States

Supporting Information

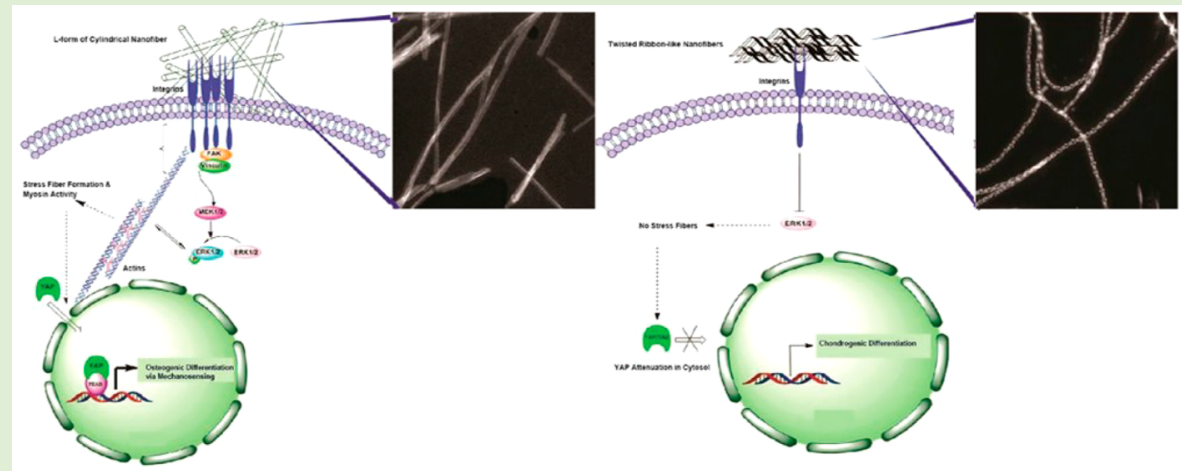

ABSTRACT: Chirality and morphology are essential factors for protein function and interactions with other biomacromolecules. Extracellular matrix (ECM) proteins are also similar to other proteins in this sense; however, the complexity of the natural ECM makes it difficult to study these factors at the cellular level. The synthetic peptide nanomaterials harbor great promise in mimicking specific ECM molecules as model systems. In this work, we demonstrate that mechanosensory responses of stem cells are directly regulated by the chirality and morphology of ECM-mimetic peptide nanofibers with strictly controlled characteristics. Structural signals presented on L-amino acid containing cylindrical nanofibers $(\mathrm{L}-\mathrm{VV})$ favored the formation of integrin $\beta 1$-based focal adhesion complexes, which increased the osteogenic potential of stem cells through the activation of nuclear YAP. On the other hand, twisted ribbon-like nanofibers (L-FF and D-FF) guided the cells into round shapes and decreased the formation of focal adhesion complexes, which resulted in the confinement of YAP proteins in the cytosol and a corresponding decrease in osteogenic potential. Interestingly, the D-form of twisted-ribbon like nanofibers (D-FF) increased the chondrogenic potential of stem cells more than their L-form (L-FF). Our results provide new insights into the importance and relevance of morphology and chirality of nanomaterials in their interactions with cells and reveal that precise control over the chemical and physical properties of nanostructures can affect stem cell fate even without the incorporation of specific epitopes.

\section{INTRODUCTION}

In their native microenvironment, cells respond to a broad range of extracellular matrix (ECM) signals by modulating their mechanical properties through cytoskeletal remodeling. ${ }^{1}$ The ECM provides a physical scaffold that is integral for the transduction of biochemical and biomechanical signals necessary for proper functioning of cells and tissues. Structural and chemical features of ECM elements are essential for their ability to elicit specific cellular responses, and synergistic interactions between these signals are also crucial for the regulation of cellular behavior. For example, integrin-binding motifs such as GFOGER occur on collagen and only exhibit

Received: June 1, 2017

Revised: August 21, 2017

Published: August 25, 2017 
bioactivity when presented within a triple helix structure. ${ }^{2}$ Thus, the macromolecular structure can substantially alter the context of even well-established biochemical signals by modulating their interactions with cellular receptors. ${ }^{3}$

Stem cells adapt environmental signals as biochemical information through contractile forces acting on fibronectin fibrils. ${ }^{4}$ In addition, fibrillar proteins in the ECM exhibit certain structural fingerprints that impart them with their fundamental functions. For example, different collagen types in various supramolecular structures, including geometric networks, membrane-spanning fibrils, and beaded-filaments, contribute to a diverse range of functions such as providing tensile strength and enabling resistance to plastic deformation and rupture. ${ }^{5}$ In addition, collagen fibrils exhibit a periodicity known as the D-band, which determines the stiffness of the microenvironment. Alterations in this periodicity are known to result in disease due to disorders in the shape and mechanical strength of the fibrillar structure. ${ }^{6}$

Supramolecular structures of the ECM fibrillar proteins are tissue-specific and their mechanical properties are optimized to react to the specific range of external and internal forces that is routinely experienced by each tissue. Through these proteins, externally applied mechanical forces function at the molecular level by regulating focal adhesion (FA) point size, shape, and composition in cells. ${ }^{7}$ In addition to supramolecular structures, chirality is also a characteristic of many biomacromolecular interactions that govern cellular behaviors. ${ }^{8-10}$ In fact, a wide range of biomacromolecules function precisely due to specific enantiomeric interactions that fail to occur if both partners do not share a chiral configuration. ${ }^{11}$ If any particular component was to be replaced by an unsuitable enantiomeric counterpart, the function of the whole system would be lost due to the ensuing destabilization effect that distorts the secondary structure of proteins and other biomacromolecules. ${ }^{12}$

Inspired by the features of tissues and their ECM, a broad range of functional therapeutic biomaterials have been developed by emulating the physical, chemical and biological properties of native tissue microenvironments. ${ }^{13-15}$ Among these biomaterials, peptide nanofibers have great potential in mimicking natural ECM by incorporating specific signal sequences. ${ }^{16,17}$ While the biological activity of peptide nanofibers is typically based on the cellular recognition of their functional epitope sequences, their physical and chemical properties are also essential for mediating cell-biomaterials interactions. ${ }^{7}$ Hence, self-assembled supramolecular peptide amphiphile nanofibers provide a useful toolkit for the investigation of cell-ECM interactions by exhibiting a diversity of mechanical properties despite their simple, well-defined, and highly bioactive structures. ${ }^{18-20}$ Self-assembly can be triggered through different external and internal forces while providing an extremely large morphological diversity and various physical and chemical features as a result. ${ }^{21,22}$ Several studies have demonstrated that nanofibers with distinct morphology and chirality are able to selectively interact with cells to elicit specific cellular responses, but these studies only focus on one of these features in isolation from the others. ${ }^{6,8,9,23}$ However, emulating the complexity inherent to native tissues requires sophisticated biomaterials design integrating distinctive features to precisely regulate the distribution of cells, macromolecules, and structural elements at various scales and dimensions. ${ }^{24}$

In this work, we demonstrated peptide nanofibers with strictly controlled morphology and chirality to study the effects of these features on the cellular fate at the molecular and cellular levels. We monitored the control of cellular responses, including the differentiation of stem cells, by tuning the physical and chemical cues provided by the peptide nanofiber materials. Twisted ribbon-like nanofibers (L-FF and D-FF) reduced cellular spreading as well as ERK/MAPK pathway activity, resulting in genetic regulation through the repression of nuclear YAP (Yes-associated protein) activity, which reduced the osteogenic differentiation of stem cells while favoring chondrogenesis by altering cell shape through matrix-induced cellular rounding. In contrast, the L-amino acid containing cylindrical nanofibers (L-VV) produced a greater mechanical feedback and enhanced cellular spreading through increased interaction with the integrin $\beta 1$ receptor. Stem cells on L-VV scaffolds activated the ERK/MAPK pathway, which resulted in YAP/TAZ activation and nuclear YAP localization, which further increased the osteogenic differentiation of stem cells. These results demonstrate the importance of morphology and chirality on effect of ECM-mimetic nanofibers on stem and somatic cell behavior and show that the behavior of stem cells could be tuned even without the incorporation of biologically relevant epitopes.

\section{EXPERIMENTAL SECTION}

Materials. 9-Fluorenylmethoxycarbonyl (Fmoc) and tert-butoxycarbonyl (Boc) protected amino acids, $\left[4-\left[\alpha-\left(2^{\prime}, 4^{\prime}\right.\right.\right.$-dimethoxyphenyl) Fmoc aminomethyl]enoxy] acetamidonorleucyl-MBHA resin (Rink amide MBHA resin), Fmoc-Glu(OtBu)-Wang resin, and 2(1H-benzotriazol-1-yl)-1,1,3,3-tetramethyluronium hexafluorophosphate (HBTU) were purchased from NovaBiochem and ABCR. Cover glasses and tissue culture plates (24-well) were purchased from Deckglaser and BD. All other chemicals and materials used were analytical grade and obtained from Invitrogen, Fisher, Merck, Alfa Aesar, and Sigma-Aldrich. Chondrogenic differentiation media were purchased from Sigma. Live/Dead Assay (L3224), Alamar Blue, and other cell culture materials were purchased from Invitrogen. Western blotting, flow cytometry, and immunocytochemistry antibodies were purchased from Abcam, Millipore, or Santa Cruz Biotechnologies: antivinculin antibody, (Abcam, ab18058), anti-FAK antibody (Abcam, ab72140), mouse monoclonal IgG2a (Abcam, ab170191), goat antimouse IgG H\&L (Alexa Fluor 488) (Abcam, ab150113), antiIntegrin $\alpha 2$ antibody [EPR17338]-C-terminal (Abcam, ab181548), anti-FAK antibody [63D5] (Abcam, ab72140), antiphospho-FAK (Tyr397) antibody clone EP2160Y (Millipore, 04-974), anti-ERK1 + ERK2 antibody [IL-13] (Abcam, ab130004), anti-ERK1 (pT202/ pY204) + ERK2 (pT185/pY187) antibody [MAPK-YT] (Abcam, ab50011), anti-MEK1 + MEK2 antibody (ab178876) or antiphosphoMEK1 (Ser218/222)/MEK2 (Ser222/226) antibody (Millipore, 05747), Goat Anti-Mouse IgG H\&L (Alexa Fluor 488) (Abcam, ab150113), YAP antibody (H-9) (SCBT, sc-271134).

Synthesis and Characterization of Peptide Amphiphile Molecules. All peptides were synthesized by using Fmoc solid phase peptide synthesis. All peptides including lauric acid were constructed on Fmoc-Rink Amide MBHA resin. Amino acid coupling reactions were performed with 2 equiv of Fmoc-protected amino acid, 1.95 equiv of HBTU, and 3 equiv of DIEA for $2 \mathrm{~h}$. The Fmoc protecting group removal was performed with $20 \%$ piperidine/DMF solution for $25 \mathrm{~min}$. Cleavage of the peptides from the resin was carried out with a mixture of trifluoreacetic acid (TFA)/triisopropylsilane (TIS) $/ \mathrm{H}_{2} \mathrm{O}$ at a ratio of $95: 2.5: 2.5$ for $2 \mathrm{~h}$. Excess TFA was removed by rotary evaporation. The remaining peptide was triturated with ice-cold diethyl ether, and the resulting white precipitate was freeze-dried. All peptides were purified by preparative liquid chromatography (Prep-HPLC), and positively charged peptides were treated with $1 \mathrm{mM} \mathrm{HCl}$.

Before each characterization, 1\% (w/v) EE-PA solutions and KK-PA solutions were dissolved in water separately. Four samples, L-VV, DVV, L-FF, D-FF, which are listed in Table 1, were prepared by mixing 
Table 1. List of Positively and Negatively Charged Peptide Amphiphiles

\begin{tabular}{cll} 
networks & \multicolumn{1}{c}{ positive PA } & \multicolumn{1}{c}{ negative PA } \\
L-VV & Lauryl-VVAGKK-Am & Lauryl-VVAGEE-Am \\
D-VV & Lauryl-vvaGkk-Am & Lauryl-vvaGee-Am \\
L-FF & Lauryl-FFAGKK-Am & Lauryl-FFAGEE-Am \\
D-FF & Lauryl-ffaGkk-Am & Lauryl-ffaGee-Am \\
\hline
\end{tabular}

positively and negatively charged peptide solution pairs at 1:1 volume ratio (Table 1). These neutralized samples were incubated overnight and all characterizations were done at the physiological $\mathrm{pH}$.

Liquid Chromatography and Mass Spectrometry (LC-MS). Agilent Technologies 6530 Accurate-Mass Q-TOF-MS equipped with HPLC using Zorbax SB-C8 column was used for LC-MS analysis. LC-MS sample was prepared in $0.5 \mathrm{mg} / \mathrm{mL}$ concentration. Mobile phase solutions were water $(0.1 \%$ formic acid $)$ and acetonitrile (ACN) (0.1\% formic acid). LC-MS was run for $30 \mathrm{~min}$ for each sample and it started with $2 \% \mathrm{ACN}$ and $98 \% \mathrm{H}_{2} \mathrm{O}$ for 5 min. Then gradient of ACN reached $100 \%$ in 20 min. Finally, its concentration was dropped to $2 \%$, and it was kept running for $5 \mathrm{~min}$. Solvent flow was $0.65 \mathrm{~mL} / \mathrm{min}$, and $5 \mu \mathrm{L}$ sample was injected.

Circular Dichroism (CD). A Jasco J-815 CD spectrophotometer was used for CD analysis. The $1 \%(\mathrm{w} / \mathrm{v})$ positively and negatively charged peptide solution mixtures were diluted first to $2 \mathrm{mM}$, then to $0.25 \mathrm{mM}$ concentration, gradually. This prevented the disintegration of the coassembled network through dilution. The $0.25 \mathrm{mM}$ solutions were used for the $\mathrm{CD}$ measurement in $1 \mathrm{~mm}$ quartz cell. Peptide solution was measured from 300 to $190 \mathrm{~nm}$ with 0.1 data pitch, $100 \mathrm{~nm} / \mathrm{min}$ scanning speed, $1 \mathrm{~nm}$ bandwidth, and $4 \mathrm{~s}$ D.I.T. Averages of three measurements were used, and sensitivity was selected as standard.

Transmission Electron Microscopy (TEM). Imaging of the peptide nanostructures was achieved by TEM (FEI, Tecnai G2 F30) at $100 \mathrm{kV}$. For peptide nanofiber staining, uranyl acetate solution in water $(2 \mathrm{wt}$ $\%)$ was used. Each of four $1 \%(\mathrm{w} / \mathrm{v})$ peptide stock solutions was gradually diluted to $2 \mathrm{mM}$ solution, then to $50 \mu \mathrm{M}$ TEM sample solution. These diluted samples were placed on a Lacey carbon coated copper grid. Ten microliters of diluted sample solution was dropped on a grid and kept there for $8 \mathrm{~min}$. The excess was removed by pipet. Then $20 \mu \mathrm{L}$ of 2 wt \% uranyl acetate solution was put on a parafilm sheet. The grid was placed on the top of the drop upside down and kept there for $5 \mathrm{~min}$. Stained grids were dried in a fume hood at room temperature overnight.

Small-Angle X-ray Scattering (SAXS). Experiments on single component peptide solutions were performed on beamline B21 at Diamond Light Source, Harwell, U.K. Solutions (1 or 0.5 wt \% for more viscous samples) were loaded into the 96-well plate of an EMBL BioSAXS robot. Aliquots of solutions $(25 \mu \mathrm{L})$ were then injected via an automated sample exchanger at a slow and very reproducible flux into a quartz capillary (1.8 $\mathrm{mm}$ internal diameter) in the X-ray beam. For D-VVEE, the 1 wt \% sample was too viscous to flow into the capillary; however, dilution to $0.5 \mathrm{wt} \%$ enabled sample delivery into the beam. The quartz capillary was enclosed in a vacuum chamber to avoid parasitic scattering. After the sample was injected in the capillary and reached the X-ray beam, the flow was stopped during the SAXS data acquisition. SAXS frames were collected with duration of 20 or $100 \mathrm{~s})$. B21 operated with a fixed camera length $(4.01 \mathrm{~m})$ and fixed energy $(12.4 \mathrm{keV})$. The images were captured using a Pilatus $2 \mathrm{M}$ detector. Data processing (background subtraction, radial averaging) was performed using the dedicated beamline software Scatter.

SAXS data were modeled using the software SASfit ${ }^{25}$ with model "Bilayer Gauss". This model describes tape-like structures represented as bilayers with electron dense cores and lower electron density surfaces. The model, used in several previous papers, ${ }^{26,27}$ was that of Pabst et al. $^{28}$ In some cases (data for L-FFEE and D-FFKK), an alternative nanotube form factor provided a better fit to the data at low $q$ where a maximum was observed in the intensity, corresponding to helically wrapped nanotapes. The fitting was done using SASfit. ${ }^{25} \mathrm{~A}$ flat background was added in the model for all data.
Simultaneous SAXS/WAXS experiments on gel-forming mixtures were performed on beamline BM26B at the ESRF. Samples were placed in DSC pans modified with mica windows to enable transmission of the X-ray beam. The sample to SAXS detector distance was $3.16 \mathrm{~m}$ using a wavelength of $1.033 \AA$ A . A Dectris-Pilatus 1 $\mathrm{M}$ detector with a resolution of $981 \times 1043$ pixels and a pixel size of $172 \times 172 \mu \mathrm{m}^{2}$ was used to acquire the 2D SAXS scattering patterns. Standard corrections for sample absorption and background subtraction were performed. The data were normalized to the intensity of the incident beam (to correct for primary beam intensity fluctuations) and were corrected for absorption and background scattering. Diffraction from silver behenate was used to calibrate the wavevector scale of the scattering curve.

Molecular Dynamics Simulations. All-atom explicit solvent simulation systems were prepared for four PA nanofibers. Each PA nanofiber system was constructed using 18 layers that were composed of 12 PAs. The starting configuration for each PA nanofiber was done based on previous simulations, such that 19 layer with $12 \mathrm{PA}$ in each layer configuration gave rise to the most stable configuration for PAs having similar length compared with PAs given in this work. ${ }^{29}$ To maintain 1:1 stoichiometry between glutamate (GLU-G) and lysine (LYS-K) having peptides, adjacent layers were constructed with either GLU only or LYS only peptides. To establish ionic interactions between the main simulation box and periodic images, 18 layers were chosen instead of 19 layers. Each layer was built by placing 12 PAs with $30^{\circ}$ angle separation. Adjacent layers were put together with $5 \AA$ distance away and $15^{\circ}$ angle rotation (Figure S8). The PA nanofibers were solvated with TIP3 water molecules and, $\mathrm{Na}^{+}$and $\mathrm{Cl}^{-}$ions were added to reach $0.15 \mathrm{M}$ salt concentration. Resulting simulation system boxes contained around 120000 atoms.

MD simulations for the PA nanofibers were performed using NAMD program (version 2.9) with CHARMM force field. ${ }^{30,31}$ Prior to production simulations, simulation systems were minimized with 1000 minimization steps. Production simulations of $100 \mathrm{~ns}$ were carried out for each PA nanofiber system at $1 \mathrm{~atm}$ pressure and $310 \mathrm{~K}$ temperature. Electrostatic interactions were calculated using the particle-mesh Ewald method with a grid spacing. ${ }^{32}$ The cutoff for van der Waals interactions was taken as $12 \AA$ with a switching function after $10 \AA$. Simulation trajectories were integrated with a time step of 2 fs, with all interactions calculated at every time step. Atomic coordinates were collected every 10 ps. The analyses were applied to the last $20 \mathrm{~ns}$ of each trajectory. Hydrogen bonds and radial distribution functions (RDF) were calculated using CPPTRAJ program. ${ }^{33}$ Nonbonded interaction energy calculations on simulation trajectories were carried out using VMD program. ${ }^{34}$

Nanomechanical Characterization of Peptide Nanofibers by Atomic Force Microscopy (AFM). For AFM measurements, peptide nanofibers were prepared as $5 \mu \mathrm{M}$ in same method with TEM imaging protocol and were dropped onto a freshly cleaved mica surface. Silicon nitride (Budget Sensors) AFM probes were used for contact mode imaging of the self-assembled peptide nanofibers in liquid. For performing of force mapping in contact-mode, Asylum Research MFP3D AFM was used. Force maps were taken at a resolution of $32 \times 32$ from a $1-5 \mu \mathrm{M}$ area. A trigger point of $0.5 \mathrm{~V}$ was applied for curve measurements. Vertical deflection correction was performed before starting force map measurements. A total of more than 800 force curves were analyzed per peptide nanofiber group for AFM analysis. The Hertz model was applied to calculate elastic moduli from approach curves. The Poisson ratio of the nanofibers was assumed to be 0.33 .

Oscillatory Rheology Analysis. Oscillatory rheology measurements were performed with an Anton Paar Physica MCR301 system. A 25 $\mathrm{mm}$ parallel plate with a gap distance of $0.5 \mathrm{~mm}$ was used at $25^{\circ} \mathrm{C}$ for all measurements. The total gel volume was adjusted as $250 \mu \mathrm{L}$. PA solutions were freshly prepared as $10 \mathrm{mM}$ and sonicated for $30 \mathrm{~min}$. Gels were prepared using the combinations seen in Table S1. The negatively charged PAs were first loaded at the center of the stage and mixed with the positively charged PAs. The upper plate was adjusted to the $0.5 \mathrm{~mm}$ position, and the gel was incubated in this position for $15 \mathrm{~min}$ prior to measurement. For strain sweep measurements, angular 
frequency was kept constant at $10 \mathrm{rad} / \mathrm{s}$, and strain was increased between 0.1 and $100 \%$. Storage and loss moduli were recorded at each strain value. All rheology measurements were with three replicates.

Scanning Electron Microscopy (SEM) Analysis. For SEM imaging, $1 \%(\mathrm{w} / \mathrm{v})$ bulk peptide nanofiber gels were prepared on silicon wafers. Samples were dehydrated in graded ethanol solutions, starting with $20 \%$ ethanol and proceeding to absolute ethanol for $10 \mathrm{~min}$ at each step. Samples were dried with a Tourismis Autosamdri-815B critical point drier, coated with $10 \mathrm{~nm} \mathrm{Au} / \mathrm{Pd}$, and imaged with a FEI Quanta 200 FEG SEM.

In Vitro Cell Culture Experiments. Peptide amphiphile nanofiber scaffolds were prepared through the protocols used for chemical characterization experiments. Briefly, PA solutions were prepared in $\mathrm{ddH}_{2} \mathrm{O}$ at a concentration of $2 \mathrm{mM}$ and sterilized under UV for $1 \mathrm{~h}$. Then the corresponding well plates (96-well plates or 24-well plates, depending on the experiment) were coated with PA combinations (Table S1). Peptide coated plates were sealed and incubated at room temperature overnight for hydrogel consolidation before use. rMSCs (rat mesenchymal stem cells, Invitrogen S1601-100, at passage 6-8), HUVECs (HUVECs were kindly provided by Yeditepe University, Istanbul, Turkey), and primary human fibroblasts (hFib) (hFibs were kindly provided by Middle East Technical University, Ankara, Turkey) were used in in vitro cell culture experiments. All cells were initially cultured in DMEM supplemented with $10 \%$ fetal bovine serum (FBS) (maintenance medium, MT) in tissue culture plates at standard culture conditions (at $37^{\circ} \mathrm{C}$ under $5 \% \mathrm{CO}_{2}$ ). For differentiation analyses, after 1 day of incubation for cell attachment, the medium was replaced with fresh MT, chondrogenic differentiation medium (Gibco) or osteogenic medium. MT supplemented with $10 \mathrm{mM} \beta$-glycerophosphate, $0.2 \mathrm{mM}$ ascorbic acid and $100 \mathrm{nM}$ dexamethasone was used as an osteogenic medium for the osteogenic differentiation of rMSCs.

Viability Analysis. Cell cultures were prepared in 96-well plates in MT medium, and cellular viability was analyzed by Alamar Blue (Invitrogen) and Live/Dead (Life Technologies) assays. Fivethousand rMSCs, HUVECs, or hFibs were seeded onto peptide coatings and their viability was measured at $24 \mathrm{~h}, 48$ and $72 \mathrm{~h}$ by measuring the fluorescence and absorbance of the reagent by spectrophotometry for the Alamar Blue assay, and imaging the cells by fluorescence microscopy for the Live/Dead Assay.

Adhesion Analysis. Cell cultures were prepared in 96-well plates (2000 cells/well, 3 replicas for each peptide nanofiber group) in MT medium and cellular adhesion was analyzed by using adhesion medium, which is serum free MT medium including $50 \mu \mathrm{g} / \mathrm{mL}$ cyclohexamide and $4 \mathrm{mg} / \mathrm{mL}$ BSA. Before seeding, cells were incubated in adhesion medium for $1 \mathrm{~h}$ and subsequently seeded onto peptide coatings in adhesion medium. After 1 and $5 \mathrm{~h}$, wells were washed with PBS, and adherent cells were stained with Calcein Am for $20 \mathrm{~min}$. The number of adherent cells was counted using ImageJ from fluorescence microscopy images.

Proliferation Analysis. Cell cultures were prepared in 96-well plates in MT medium and cellular proliferation was analyzed by a colorimetric ELISA-based BrdU assay (Cell Proliferation ELISA, BrdU; Roche) according to the manufacturer's protocol. Briefly, 5000 cells/well were seeded onto coatings, and after 1, 3, and 5 days, BrdU assay was performed by incubating cells with BrdU labeling reagent, fixing them, and staining them with anti-BrdU-POD. A manufacturerprovided colorimetric substrate was added to the wells for the development of antibody staining, and absorbance values were analyzed by a spectrophotometer.

Spreading Analysis. Cell cultures were prepared in 24-well plates on glass cover slides in MT medium and cellular spreading was analyzed by staining cells with Phalloidine/TO-PRO-3 (Invitrogen). Images were taken by fluorescence microscopy and analyzed by ImageJ software.

Gene Expression Analysis. Cell cultures (rMSC) were prepared in 24-well plates in MT or chondrogenic differentiation medium. Gene expression analyses were performed by amplifying markers for chondrogenesis (Sox-9) and osteogenesis (Runx-2) by quantitative RT-PCR (qRT-PCR). Total RNAs of rMSCs on peptide coatings were isolated using TRIzol (Invitrogen) according to the manufacturer's instructions. Yields and purities of extracted RNA were assessed by Nanodrop 2000 (Thermo Scientific). Primer sequences were designed using Primer 3 software (Table S2). cDNA synthesis from RNA and qRT-PCR were performed using SuperScript III Platinum SYBR Green One-Step qRT-PCR Kit (Invitrogen) according to the manufacturer's protocol. Reaction conditions were briefly as follows: $55^{\circ} \mathrm{C}$ for $5 \mathrm{~min}, 95^{\circ} \mathrm{C}$ for $5 \mathrm{~min}, 40$ cycles of $95^{\circ} \mathrm{C}$ for $15 \mathrm{~s}, 60^{\circ} \mathrm{C}$ for $30 \mathrm{~s}$, and $40{ }^{\circ} \mathrm{C}$ for $1 \mathrm{~min}$, followed by a melting curve to confirm product specificity. Reaction efficiencies were evaluated for each primer set through standard curves using 5-fold serial dilutions of total RNA. For the analysis of expression, primary gene expression data were normalized by the expression level of GAPDH. A comparative $\mathrm{Ct}$ method was used to analyze the results.

Protein Expression Analyses by Flow Cytometry. Flow cytometry was performed to quantify the expression of Vinculin and FAK proteins, which are important regulators of focal adhesion and cellular mechanotransduction. Prior to flow cytometry analysis, the cells were disintegrated from peptide coatings by collagenase/trypsin treatment. The supernatant was then collected and centrifuged at $2500 \mathrm{rpm}$ for 5 min. The cell pellet was washed twice with PBS, resuspended, and fixed with $4 \%$ paraformaldehyde for $15 \mathrm{~min}$ at room temperature. The solution was then centrifuged at $2500 \mathrm{rpm}$ for $5 \mathrm{~min}$, and the cell pellet was resuspended and permeabilized in $0.2 \%$ Tween-20/PBS for $15 \mathrm{~min}$ at room temperature. A primary antibody solution (either Vinculin [anti-Vinculin antibody, (ab18058)] or FAK [anti-FAK antibody (ab72140)]) or their corresponding isotype (mouse monoclonal IgG2a (ab170191)) was prepared in 3\% BSA/PBS solution at concentrations recommended by the manufacturer (Abcam) and used to stain the cells for $1 \mathrm{~h}$. After primary antibody staining, cells were washed with permeabilizing solution, pelleted by centrifugation, and stained with secondary antibodies [goat antimouse IgG H\&L (Alexa Fluor 488) (ab150113)] for $1 \mathrm{~h}$. After washing twice with permeabilizing agent, the cells were again pelleted by centrifugation and resuspended in PBS prior to cytometry analysis. A BD Accuri C6 flow cytometer system was used for cytometry, and at least 20000 events were analyzed for the measurements. For data analysis, BD Accuri C6 software was used according to the manufacturer's instructions.

Immunoblotting Analysis of Protein Expression by Western Blotting. Western blotting analyses were performed to detect the expression levels of key proteins of mechanotransduction and the associated molecular pathways. Cells were isolated from their peptide coatings by RIPA buffer (including protease and phosphatase inhibitor cocktail), and the protein containing supernatant was removed and stored at $-80^{\circ} \mathrm{C}$. Protein concentrations were determined using BCA Protein Assay Kit. Equal amounts of proteins per lane were separated by $12 \%$ SDS-PAGE and transferred to a polyvinylidene difluoride (PVDF) or nitrocellulose membrane. The membrane was blocked with 5\% nonfat milk in TBS-T at room temperature for $2 \mathrm{~h}$ and then incubated with anti-Integrin $\alpha 2$ antibody [EPR17338]-C-terminal (Abcam, ab181548), anti-FAK antibody [63D5] (Abcam, ab72140), antiphospho-FAK (Tyr397) antibody clone EP2160Y (Millipore, 04974), anti-ERK1 + ERK2 antibody [IL-13] (Abcam, ab130004), antiERK1 (pT202/pY204) + ERK2 (pT185/pY187) antibody [MAPKYT] (Abcam, ab50011), anti-MEK1 + MEK2 antibody (ab178876), or antiphospho-MEK1 (Ser218/222)/MEK2 (Ser222/226) antibody (Millipore, 05-747) overnight at $4{ }^{\circ} \mathrm{C}$. After washing in TBS-T, the blots were incubated with the corresponding horseradish-coupled secondary antibody (goat antirabbit IgG or goat antimouse IgG). The bands were visualized using Clarity Western ECL blotting substrate. GAPDH (Millipore) was used as the internal control and treated with the same protocol. Protein amounts in each sample were quantified using ImageJ software.

Immunocytochemical Analyses of Protein Detection by Confocal Microscopy. The rMSCs on peptide coatings were fixed in $4 \%$ paraformaldehyde/PBS for $10 \mathrm{~min}$ and permeabilized in $0.1 \%$ Triton $\mathrm{X}-100$ for $15 \mathrm{~min}$. For blocking, samples were incubated with 3\% (w/ v) bovine serum albumin/PBS for $30 \mathrm{~min}$ and treated with either YAP antibody (H-9) (SCBT, sc-271134) or anti-Vinculin antibody [SPM227] (ab18058) overnight at $4{ }^{\circ} \mathrm{C}$. Cells were then washed 
A

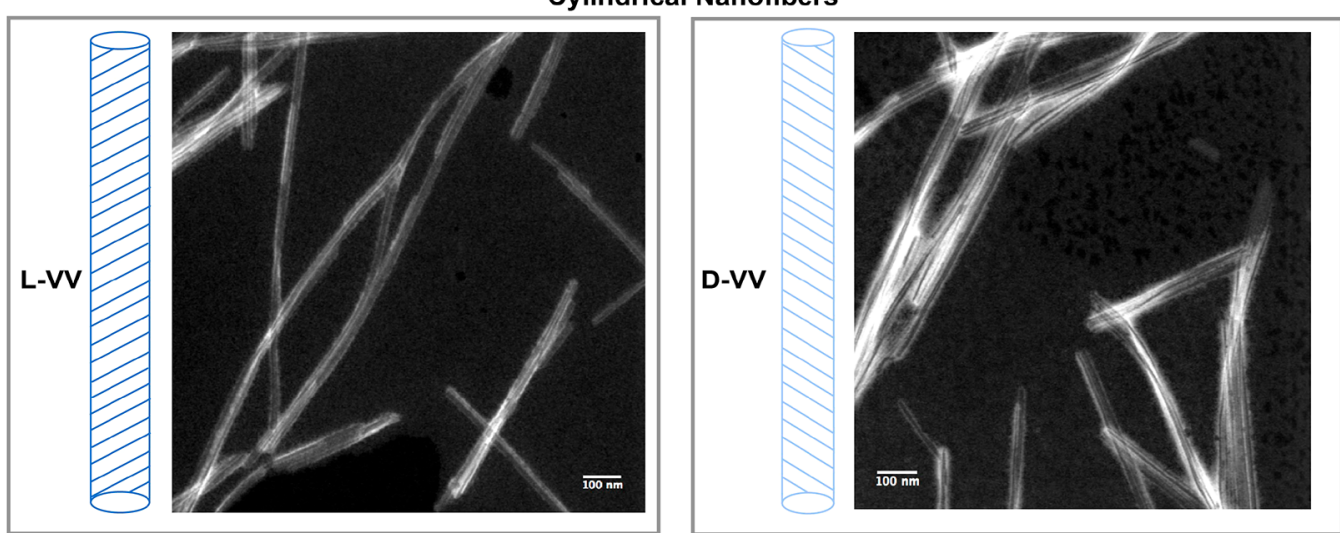

Twisted Ribbon-like Nanofibers
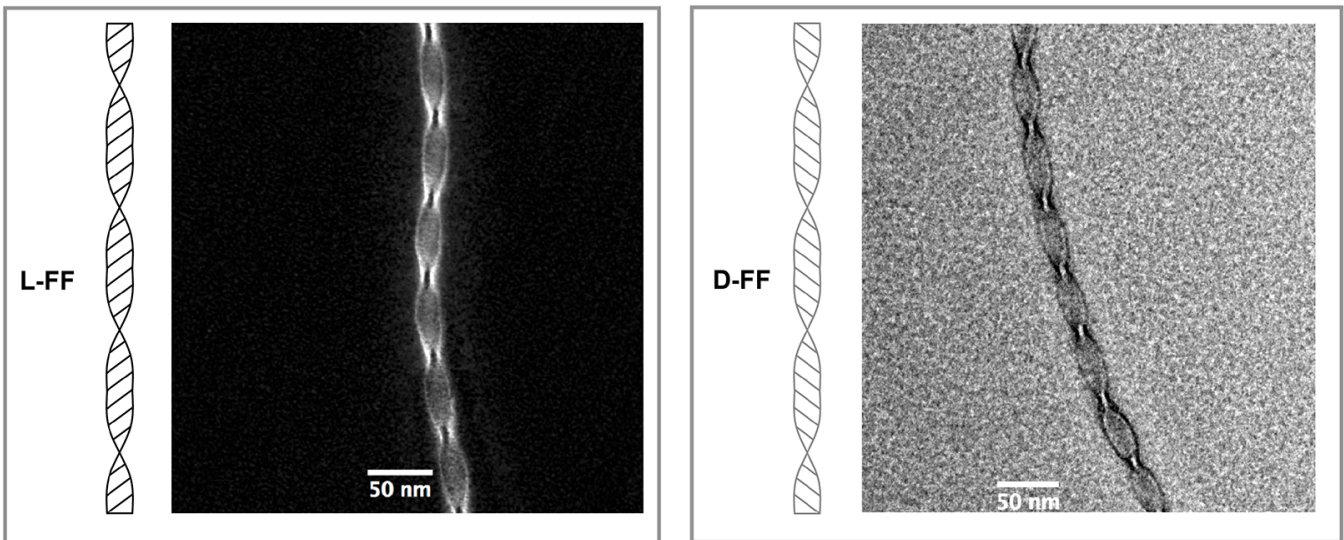

B
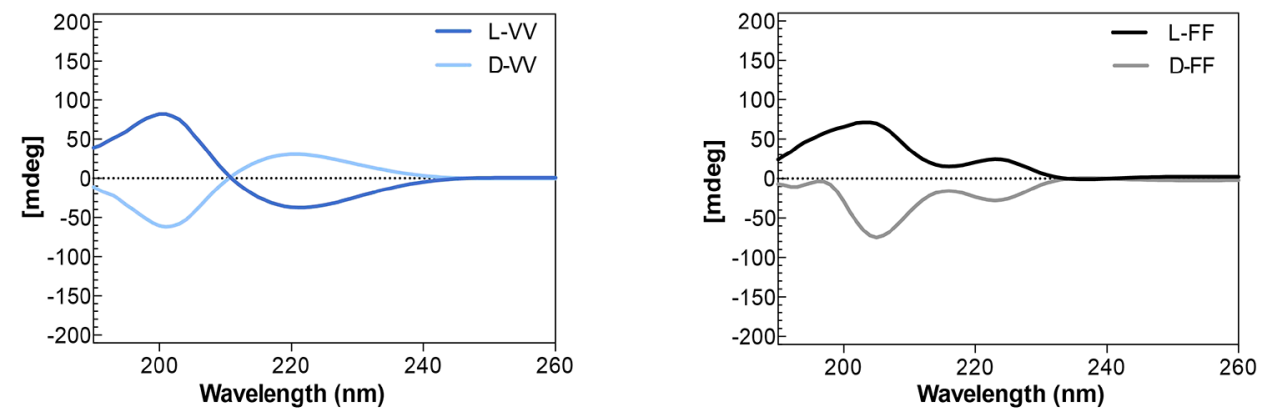

C
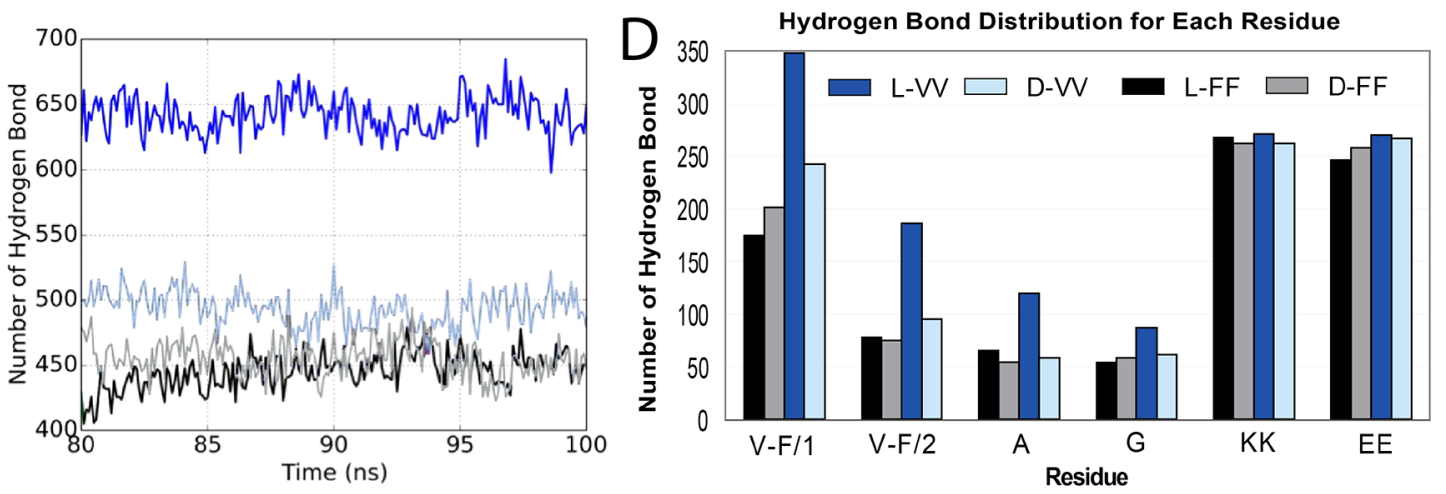

Figure 1. Structural and morphological characterization and molecular dynamics simulations of peptide nanofibers. (A) Schematic representations of peptide nanofibers and their corresponding STEM images, scale bar $=100 \mathrm{~nm}$ (L-VV and D-VV), scale bar $=50 \mathrm{~nm}$ (L-FF and D-FF). (B) Circular dichroism spectra of peptide nanofibers. (C, D) Molecular dynamics analysis of peptide nanofibers. Hydrogen bonds are determined by the following criteria: when distance between donor and acceptor atoms are lower than $3.5 \AA$, and donor-hydrogen-acceptor angle is greater than $150^{\circ}$. (C) Time series of hydrogen bond number for the last $20 \mathrm{~ns}$ of the simulations. (D) Number of hydrogen bonds that each residue forms: V-F/1 denotes the first valine or phenylalanine (numbering starts from the lipophilic tail) on the corresponding PA; V-F/2 denotes second V or F, KK and EE denote merged results for two lysine and two glutamic acid residues, respectively. L-VV, Lauryl-VVAGEE-Am/Lauryl-VVAGKK-Am; D-VV, Lauryl-vvaGeeAm/Lauryl-vvaGkk-Am; L-FF, Lauryl-FFAGEE-Am/Lauryl-FFAGKK-Am; D-FF, Lauryl-ffaGee-Am/Lauryl-ffaGkk-Am. 
with $\mathrm{PBS}$ and incubated for $1 \mathrm{~h}$ at room temperature with goat antimouse IgG H\&L (Alexa Fluor 488). All samples were counterstained with $1 \mu \mathrm{M}$ TO-PRO-3 (Invitrogen) in PBS for $20 \mathrm{~min}$ at room temperature and mounted with Prolong Gold Antifade Reagent (Invitrogen). Negative controls were obtained by omitting the primary antibody and incubating with $3 \%$ normal goat serum/PBS. Samples were imaged by confocal microscopy (Zeiss LSM510) and analyzed by ImageJ program for cell counting to determine the cell amount with nuclear YAP localization.

Collagen Adsorption Analyses by ELISA. For the determination collagen I adsorption on peptide nanofibers, indirect-ELISA was performed. ELISA plates were coated with peptide nanofibers. On the next day, plates were washed by washing buffer, dried by tapping, blocked with assay buffer (Life Technologies, DS98200) (2 h), and Collagen I (Millipore, 08-115) was added onto peptide coatings for overnight at $4{ }^{\circ} \mathrm{C}$. The next day, plates were washed by washing buffer, dried by tapping, and then they were incubated with anticollagen I (1:500) (ab6308) primary antibody for overnight at $4{ }^{\circ} \mathrm{C}$. After that, plates were washed five times with washing buffer and dried by tapping between each consecutive step. The HRP-conjugated anti-IgG antibody was used as a secondary antibody and incubated for $2 \mathrm{~h}$. The TMB (3,3',5,5'-tetramethylbenzidine) substrate was added at the last step, and the reaction was stopped after $15 \mathrm{~min}$ with stop solution (1.8 $\mathrm{N} \mathrm{H}_{2} \mathrm{SO}_{4}$ ). Color formation was measured by using a microplate reader (Spectramax M5, Microplate reader) as absorbance at $450 \mathrm{~nm}$ wavelength (reference absorbance measured at $650 \mathrm{~nm}$ and subtracted from absorbance at $450 \mathrm{~nm}$ ). All treatments were performed with at least four replicates, and peptide nanofiber coatings without collagen I incubation were used as blank.

Statistical Analysis. All data are presented as mean \pm s.e.m. (standard error of mean). All experiments were performed in at least three replicates. The significance of differences between groups was determined with either one-way or two-way analysis of variance (ANOVA) with Bonferroni's post hoc test. Differences were considered significant at $* p<0.05$, except where noted.

\section{RESULTS}

Design, Preparation and Characterization of SelfAssembled PA Molecules. Here we designed four different peptide nanofibers with distinct morphology and chirality and evaluated their interactions with cells at the molecular level. Each nanofiber consisted of two oppositely charged peptide amphiphile (PA) molecules. To obtain four peptide nanofiber types (cylindrical L-form, cylindrical D-form, ribbon-like L-form, ribbon-like D-form), eight peptide amphiphile molecules, each consisting of a hydrophobic region, a $\beta$-sheet forming region, and a hydrophilic (or charged) region were designed and synthesized in two different chiral forms (Table 1). The hydrophobic region contained a lauric acid alkyl tail that is long enough to trigger specific nanofiber organization in water. ${ }^{13}$ Either two glutamic acid or two lysine residues were used for the hydrophilic region. Four PA molecules were synthesized with a charge of +2 , and another four of them with a charge of -2 at the physiological conditions (Table 1). Two oppositely charged PA molecules coassembled into high-aspect-ratio nanostructures through electrostatic interactions between positively and negatively charged building blocks to form each nanofiber network. ${ }^{35}$ In addition, hydrophobic and hydrophilic regions of each PA molecule were linked together with a hydrogen bonding peptide region, which was essential for modulating self-assembly behavior to form cylindrical or ribbon-like structures. The valine-valine sequence caused the highest propensity for forming $\beta$-sheets for cylindrical nanofiber formation. $^{36,37}$ The phenylalanine-phenylalanine sequence produced a twisted ribbon morphology through $\pi-\pi$ stacking, which is the major promoting factor of the twisted morphology in the coassembled network for ribbon-like nanostructure formation. ${ }^{38}$ In addition, for achieving chiral differences in networks, all peptides were synthesized in both $\mathrm{L}-$ and $\mathrm{D}$ enantiomeric forms. ${ }^{39}$ Successful synthesis and purification of all eight PAs were confirmed by prep-HPLC and LC-MS (Figures S1 and S2). Morphology of the PA nanostructures was investigated by transmission electron microscopy (TEM) (Figure 1A). While L-VV and D-VV produced cylindrical nanofibers, twisted ribbon morphologies were observed for LFF and D-FF. Uniform width and pitch sizes were obtained for L-FF and D-FF. The L-FF ribbons had $21.21( \pm 3.7) \mathrm{nm}$ average diameters and $55.70( \pm 8.6) \mathrm{nm}$ average pitch size, while D-FF formed ribbons that had an average diameter of $18.21( \pm 2.7)$ $\mathrm{nm}$ and pitch size of $48.21( \pm 7.3) \mathrm{nm}$ (Figures S3 and S4). SAXS analysis showed that the form factor features of the eight PA solutions were characteristic of nanotape structures. SAXS data with model form factor fits are shown in Figure S6. The fits exhibited layer thicknesses of $38 \pm 2 \AA$ for $\mathrm{L}-\mathrm{VV}$ and $\mathrm{D}-\mathrm{VV}$ samples and $29 \pm 1.5 \AA$ for L-FF and D-FF samples. This is consistent with completely interdigitated bilayer structures (i.e., fully overlapped lipid chains in the bilayer interior) since the length of a PA molecule packed in a parallel $\beta$-sheet is estimated to be $37 \AA$. For all four gels, SAXS showed very similar form factors (Figure S7) that exhibited features of twisted ribbons.

Hydrogen-bonding pattern and secondary structures of the networks were characterized by circular dichroism (CD) spectroscopy. For L-VV and D-VV ensembles, $\beta$-sheet secondary structure was detected by fingerprint bands at 221 and $200 \mathrm{~nm}$ (Figure 1B) corresponding to $\mathrm{n}-\pi^{*}$ and $\pi-\pi^{*}$ transitions, respectively. ${ }^{40} \mathrm{CD}$ spectra of L-FF and D-FF ensembles were more complicated, presumably a combination of absorptions of $\beta$-sheet motif and phenylalanine residues. In both cases, the CD spectra of $\mathrm{L}$-form and $\mathrm{D}$-form peptide nanofibers were mirror images of one another due to their opposite chirality. In the case of phenylalanine-containing L-FF and D-FF ensembles, a deviation was observed from perfect $\beta$-sheet structure because $\pi-\pi$ interactions between aromatic side chains caused building blocks to assemble not with a $90^{\circ}$ angle to the elongation axis, as with $\mathrm{L}-\mathrm{VV}$ and $\mathrm{D}-\mathrm{VV}$, but with a narrower angle that rotated and twisted across the length of the fiber. This rotation in conformation has been reported as a twisted $\beta$-sheet secondary structure. ${ }^{36}$ In twisted assemblies, relatively weaker hydrogen bonds are formed due to the increases in bond length. Therefore, a red shift was observed in the CD spectrum (Figure 1B). To get an estimate on contribution of phenylalanine residues, spectra of $\mathrm{L}-\mathrm{VV}$ and $\mathrm{D}-\mathrm{VV}$ ensembles were subtracted from spectra of $\mathrm{L}-\mathrm{FF}$ and $\mathrm{D}-\mathrm{FF}$ ensembles, respectively. CD difference spectra (Figure S5) are indicative of the contribution of the phenylalanine residues to higher energy transitions $\left(\mathrm{n}-\pi^{*}\right.$ and $\left.\pi-\pi^{*}\right)$ of the amide group. The CD difference spectrum between L-isomer ensembles showed two positive peaks at 222 and $208 \mathrm{~nm}$ and a negative peak at $198 \mathrm{~nm}$, which resemble CD spectrum of $N$-acetyl-L-phenylalanine amide. ${ }^{41}$

All-atom explicit solvent classical molecular dynamics simulations were performed to investigate the molecular organization of the PAs at the atomic level (Figure S8-S11). Time series of the nonbonded interactions within peptide structures are shown in Figure S9A. Total nonbonded energies of L-FF and D-FF peptide nanofibers are similar to each other and higher than D-VV and L-VV nanofibers. On the other hand, L-VV nanofibers have lower nonbonded interaction energy than their D-VV counterparts. When only van der Waals interactions 
are considered, phenylalanine- and valine-containing PAs show different profiles. $\mathrm{L}-\mathrm{VV}$ and $\mathrm{D}-\mathrm{VV}$ nanofibers have lower energies, whereas L-FF and D-FF nanofibers have more van der Waals interactions. To elucidate this behavior, we calculated only the nonbonded interaction energies between atom pairs composed of the side-chains of phenylalanine and valine amino acids (Figure S9B). We observed a high difference in the van der Waals interaction energy between peptide nanofibers having phenylalanine and valine residues. This suggests that van der Waals energy difference between nanofibers containing phenylalanine and valine residues is due to high repulsion between phenylalanine side-chain atoms. It is also important to note that even though phenylalanine residues form favorable electrostatic interactions compared to valine residues, and the total nonbonded interaction energy is dominated by van der Waals interactions (Figure S9). High van der Waals repulsion between phenylalanine residues plays a critical role in the formation of twisted nanofibers. Even though the time scale of the simulations is not long enough to observe twisting in phenylalanine-containing nanofibers, energy analysis shows the driving force for formation of twisted nanofibers. Hydrogen bond analysis for the peptide nanofibers (Figure 1C) shows the number of unique interpeptide $\mathrm{H}$-bond in each nanofiber as a function of simulation time. Phenylalanine-containing nanofibers have the lowest number of $\mathrm{H}$-bonds; the number of $\mathrm{H}$ bonds in D-FF and L-FF nanofibers fluctuates around 450. In contrast, the $\mathrm{H}$-bond number in valine-containing peptide nanofibers increases up to 500 in $\mathrm{D}-\mathrm{VV}$ and 650 in $\mathrm{L}-\mathrm{VV}$ nanofibers. This difference in $\mathrm{H}$-bond number between valine and phenylalanine-containing nanofibers suggests that bulky aromatic phenylalanine side-chains could limit the H-bond formation ability of phenylalanine-containing nanofibers. Interestingly, the number of $\mathrm{H}$-bonds in $\mathrm{D}-\mathrm{VV}$ is much lower than the number of $\mathrm{H}$-bonds in $\mathrm{L}-\mathrm{VV}$ nanofiber. The contribution of each amino acid to the H-bond number is shown in Figure 1D. Individual contribution of amino acids in phenylalanine-containing nanofibers does not differ considerably in L- and D-enantiomers. However, all amino acids except $\mathrm{K}$ and $\mathrm{E}$ residues in valine-valine containing nanofibers have more H-bonds in L-form than in D-form. The two valine residues contribute substantially to the $\mathrm{H}$-bond number difference between L- and D-forms of valine-containing PAs. Therefore, we investigated conformational and dynamic behavior of valine residues in $\mathrm{L}-$ and $\mathrm{D}$-form to understand the $\mathrm{H}$-bond number differences in valine residues. Valine residues sterically hinder $\mathrm{H}$-bond formation in D-form as shown in radial distribution function (RDF) analysis. Figure S10 demonstrates atom-pair distribution as a function of pairdistance for the atom-pairs between valine side-chain gamma carbons and valine backbone oxygen, and the atom-pairs between valine side-chain gamma carbons and hydrogen atom bound to amide nitrogen. In these plots, the valine side-chain gamma carbons are closer to the valine backbone in D-form compared to the L-form. This result suggests that side-chain steric hindrance plays a role in hampering H-bond formation in D-amino acid-containing nanofibers. Figure S11 shows Ramachandran plots for valine residues in $\mathrm{L}$ - and $\mathrm{D}$-nanofibers. Dihedral angles for the first valine residue in L-form populated around $\mathrm{phi}=-110, \mathrm{psi}=-135$, which is close to the $\beta$-sheet region center (a study done by Hovmöller et al. on the analysis of protein structures reports that backbone dihedrals of valine amino acid for $\beta$-sheet forming conformations populate around the center where phi $=-117.7$ and $\mathrm{psi}=127.8$ for parallel beta strands, and phi $=-121.2$ and $p s i=132.5$ for antiparallel beta strands ${ }^{42}$ ). However, dihedral angle population for the first valine in $\mathrm{D}$-form shifts to the center where phi and psi angles around -90 and -125 , respectively. Dihedrals of the second valine in L-form nanofibers are not localized at a certain region; they can sample both regions near the ideal $\beta$ sheet region and regions where phi and psi are around -140 degrees. On the other hand, dihedral angle population for the second valine in D-form PAs shifts toward the upper side of the Ramachandran plot. According to Hovmöller et al., for conformations that form random coil structures, valine dihedrals shift toward higher phi and lower psi angles. ${ }^{42}$ Hence, the dihedral angle population shift observed in our calculations for D-amino acidcontaining nanofibers could imply that backbone conformation in D-amino acids deviates from the $\beta$ sheet region toward random coils. Hence, this could affect the H-bond forming ability of $\mathrm{D}$-valine residues in PAs that form nanofibers.

Oscillatory rheology measurements were performed for mechanical analysis of the bulk gel. Time sweep, frequency sweep, and strain sweep rheology analyses were conducted to investigate the gel formation mechanics and viscoelastic properties of the peptide nanofiber networks (Figure S12). Storage moduli of all nanofiber networks were found to be higher than their loss moduli suggesting that the materials are hydrogels. In addition, we found that cylindrical nanofiber networks have higher elastic moduli than twisted ribbon-like nanofiber networks. On the other hand, we did not detect any difference in elastic moduli of nanofibers between L- and Dforms. In addition, we performed SEM analyses, which showed that the nanofiber network morphology was similar for all bulk hydrogel groups (Figure S22). For nanomechanical characterization of peptide nanofibers, elastic behavior of peptide nanofibers was investigated by atomic force microscopy (AFM) measurements (Figures S13 and S14). Force mapping measurements were employed on nanofibers and nanobundles, and elastic modulus values were calculated by fitting approach curves using the Hertz model in MATLAB (Figure S13). Both L-form of cylindrical and twisted ribbon-like nanofibers had significantly higher elastic moduli than their D-forms. L-VV nanofibers had the highest stiffness at $45.62 \mathrm{MPa}, \mathrm{D}-\mathrm{VV}$ had $15.03 \mathrm{MPa}$, L-FF had $32.04 \mathrm{MPa}$, and D-FF had 10.03 MPa. Interestingly, we observed that the difference in elastic moduli of $\mathrm{L}$ - and $\mathrm{D}$-form of VV-nanofibers is consistent with the $\mathrm{H}$ bond density according to molecular dynamic simulations.

Peptide Nanofibers Provide a Biocompatible Environment for Cell Culture. Cellular viability, adhesion, proliferation, and spreading were evaluated qualitatively and quantitatively prior to testing the effects of nanofiber morphology and chirality on cellular behavior. Viability analysis showed that all peptide nanofibers were biocompatible and conducive to the growth and proliferation of stem cells through 3 culture days (Figure S15A). Live/Dead assay also showed that few to no dead cells were present on all scaffolds, which supports the results of Alamar Blue assay and demonstrates that these peptide nanofibers are highly biocompatible (Figure S16). In addition, Alamar Blue results indicated that the morphology and chirality of the peptide nanofibers do not affect the metabolic activity of cells.

When cells are exposed to a new microenvironment, cellular processes are initially altered to mediate substrate adhesion prior to the resumption of regular metabolic pathways. At this initial step, the primary role of the nanostructure scaffold is to provide a suitable set of signals for cell attachment. Adhesion 

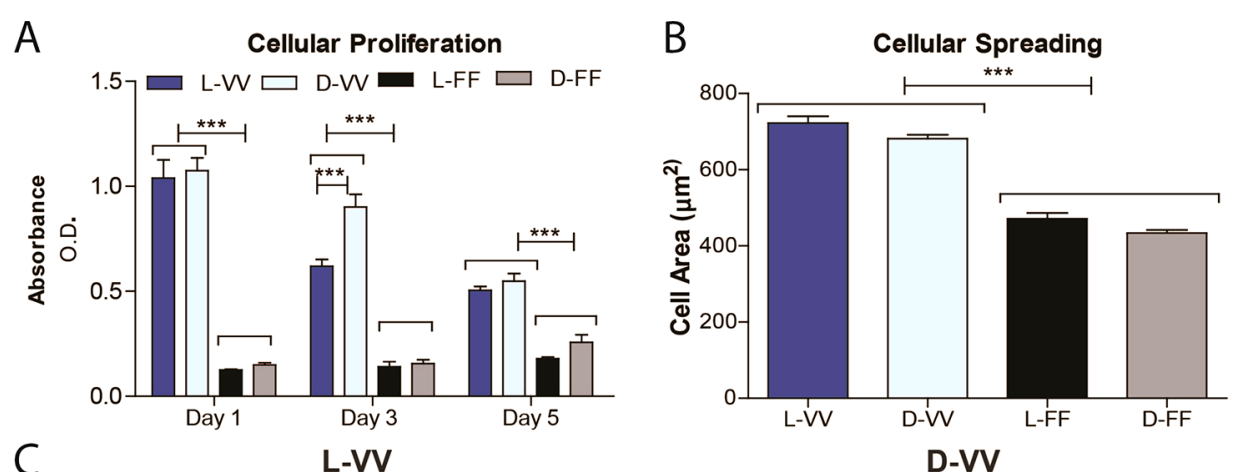

C

L-VV

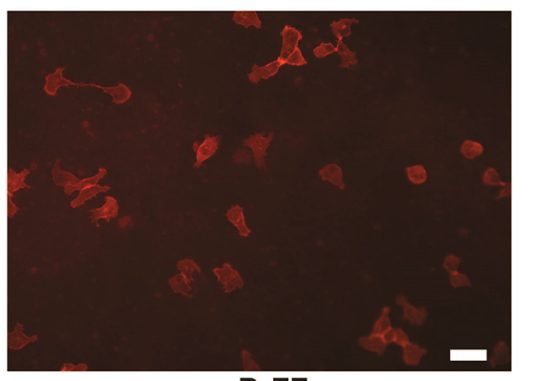

L-FF

D-FF
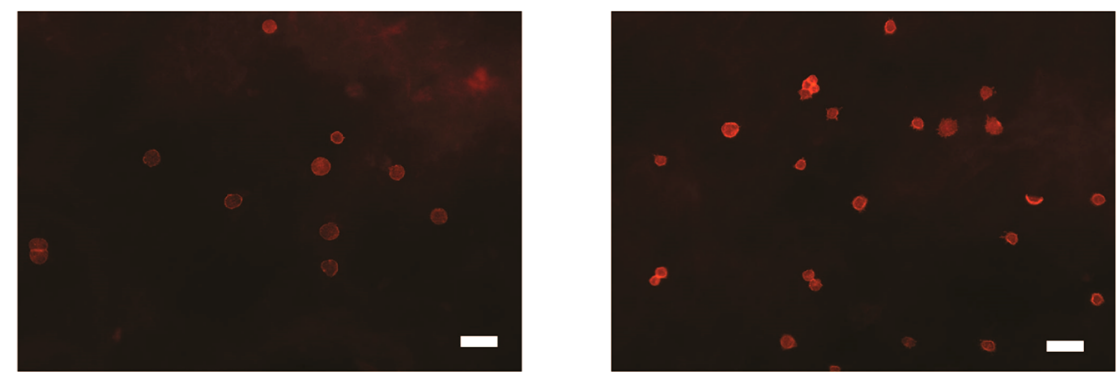

D
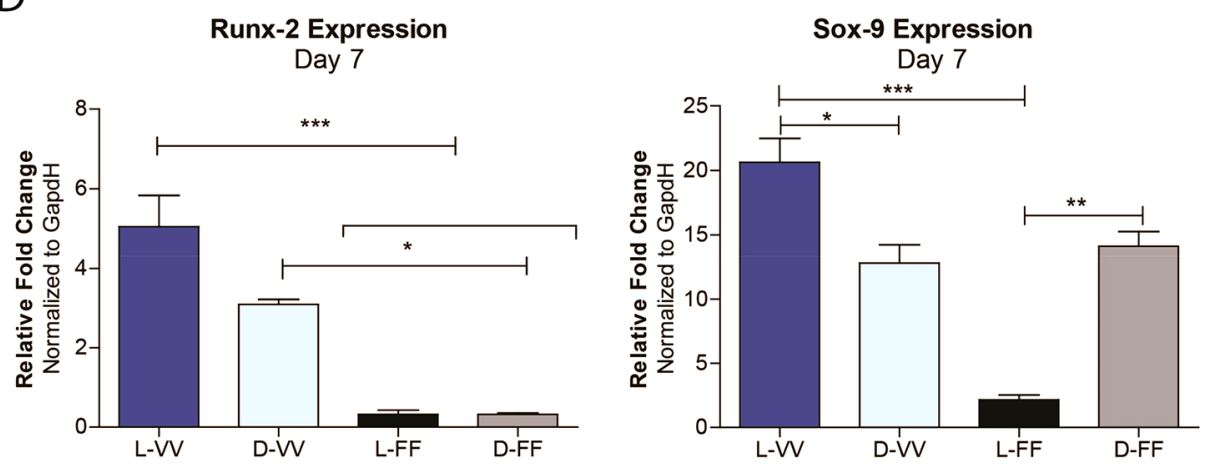

Figure 2. Cellular proliferation, spreading, and differentiation analyses of MSCs on peptide nanofibers. (A) Cellular proliferation measurement of MSCs by BrdU assay through 5 days of culture $(* p<0.05, * * p<0.01, * * * p<0.001$ by two-way ANOVA with Bonferroni's posthoc test, mean \pm s.e.m.). (B) Quantification of cellular spreading measurements of MSCs $24 \mathrm{~h}$ after seeding on peptide nanofibers, obtained by ImageJ analysis of cells stained with phalloidine and imaged by fluorescence microscopy $(n>50$ cell per group $(* p<0.05, * * p<0.01, * * * p<0.001$ by one-way ANOVA with Bonferroni's posthoc test, mean \pm s.e.m.). (C) Representative fluorescent images of MSCs $24 \mathrm{~h}$ after seeding on peptide nanofibers, scale bar $=$ $50 \mu \mathrm{m}$. (D) Osteogenic and chondrogenic differentiation analyses by qRT-PCR. Expressions of Runx-2 and Sox-9 were quantified on day 7 ( $* p<$ $0.05, * * p<0.01, * * * p<0.001$ by one-way ANOVA with Bonferroni's posthoc test, mean \pm s.e.m.).

analysis of peptide nanofiber structures showed that all peptide nanofibers facilitated the initial attachment of stem cells after 1 and $5 \mathrm{~h}$ of culturing (Figure S15B,C). When compared to tissue culture plate (TCP), cells were found to immediately attach to peptide nanofiber surfaces within the first few hours $(1-5 \mathrm{~h})$ of culture, and no significant differences were observed in the initial attachment of cells among different morphological and chiral groups.
In addition, we analyzed collagen adsorption on the peptide nanofibers to examine whether there is any differential effect of these nanofibers on the matrix protein adsorption. We found that there was no specific collagen binding on none of the nanofiber groups (Figure S21). We did not measure specific absorbance values different from blank controls (only peptide nanofibers without collagen addition), which indicated that there was no specific binding of collagen and there was no 
difference among peptide nanofiber groups. When there is specific epitope, on the other hand, there should be specific binding that should cause the significant increase in the absorbance that we measured in our previous studies by using same optimized protocol. ${ }^{66,67}$ This result showed that peptide nanofibers can adsorb proteins but interactions among them are short-term transitional interactions. If there is no specific binding epitope on the peptide amphiphile molecules, any kind of protein (such as ECM protein, growth factors, or antibodies) can interact noncovalently with peptide nanofibers regardless of their chirality or morphology. However, these interactions are not permanent and cellular adhesion is not being interrupted by these adsorptions for our case. In this respect, although all of the peptide nanofibers have similar binding capacity for collagen adsorption, we observed different level of cellular responses among peptide nanofiber groups, which we concluded that differences in cellular responses were resulted via different mechanotransduction pathways directly depending on peptide nanofiber-cell interactions. Similarly to this observation, nanoscale topography was shown to regulate collective cell function through cell adaptation mechanism largely independent of adsorbed proteins. ${ }^{68}$

In addition to stem cells, an adherent primary human cell line (HUVECs) and human fibroblasts (hFibs) were also analyzed to determine the effect of peptide nanofibers morphology and chirality on cellular responses, and both cell types were found to readily adhere to peptide scaffolds (Figure S17A,B). While HUVECs adhered significantly less on L-form of twistedribbon-like nanofibers (L-FF) among other groups, there were no significant differences between the groups for the adhesion of hFib cells (Figure S17B). The adhesion results, therefore, show the number of cells that were able to initially establish themselves on the peptide nanostructures.

Cellular Proliferation and Spreading Are Mediated by Nanofiber Morphology and Chirality. Following cellular adhesion, a bioactive scaffold should ideally provide the necessary signals for activating cellular processes such as proliferation, spreading and differentiation. According to proliferation analysis results (Figure $2 \mathrm{~A}$ ), cells demonstrated different proliferation rates on different peptide nanofibers, suggesting that nanofiber morphology and chirality play an important role in mediating nanofiber-cell interactions. Proliferation of rMSCs was tracked for 5 days, and both Land D-forms of the twisted ribbon-like nanofibers (FF) were found to elicit significantly lower proliferation of rMSCs during 5 days of incubation. After 1 day of culture, cells proliferated on L- and D-forms of the cylindrical nanofibers (VV-PA) to a much greater extent than FF-nanofiber and TCP groups. Moreover, the importance of chiral differences for cylindrical nanofibers was seen even after 3 days of culture, where proliferation rates of rMSCs on L-VV decreased much more than D-VV. After 5 days, proliferation rates of rMSCs cultured on the L- and Dforms of VV-nanofibers had decreased but were still higher than FF-nanofiber groups. Confluence is a likely reason for this effect, since L-VV and D-VV cells would stop proliferating through contact inhibition after an early increase in their population. In contrast, L-FF and D-FF do not rapidly crowd the well plate and can sustain their growth for a longer period of time but do not increase the proliferation, which is potentially due to regulation of cell differentiation by different morphological signals than VV-nanofibers, which was also evident in chondrogenesis potential of cells on FF-nanofibers. In addition, studies on stem cells also showed that for particular differentiation lineages, proliferation of stem cells were decreased. ${ }^{43}$ Overall, peptide nanofibers were found to strongly alter cellular processes even after short-term $(24 \mathrm{~h})$ culture, and proliferation on twisted ribbon-like nanofibers (FF-PAs) was limited compared to cylindrical nanofibers (VV-PAs).

After the collective response of cell cultures to different nanofibrous morphologies and chirality was analyzed, cellular areas were measured to investigate individual cellular responses (Figure 2B,C). Although neighboring cells and cellular confluence may affect the spreading of cells, each cell spreads individually through its own receptors and specific receptorligand interactions. ${ }^{44}$ Cells were seeded on all peptide nanofiber groups at the same cell concentration to avoid any initial confluency differences. We had already observed that the cells all adhered similarly (at the time of initial attachment of cells through 1-5 h) according to cellular adhesion analysis. The rMSCs cultured on L-and D-form of twisted ribbon-like nanofibers (FF-PA) exhibited significantly less cell spreading than those cultured on cylindrical nanofibers (VV-PA). In addition to rMSCs, the spreading of HUVECs and hFibs was also evaluated (Figure S16C). Among peptide nanofibers with different morphology and chirality, HUVECs on the L-form of nanofibers (L-VV) exhibited less spreading than the D-form nanofibers (D-VV). As with other cell lines, the L-FF twisted ribbons limited the spreading of hFibs to a greater extent than the D-FF nanostructures, while the D-form of VV-nanofibers was much more inhibitory than the L-form for VV-nanofibers. As such, both the morphology of nanofibers and their chirality had distinctive effects on cellular responses. Twisted ribbon-like nanofibers exhibited a more pronounced restrictive effect on cellular spreading in the L-form, whereas cylindrical nanofibers were more inhibitory in their D-form, especially for fibroblasts. Although we observed similar mechanotransductive responses on peptide nanofibers for different cell types, to elaborate on the inherent effect of designed peptide nanofibers for future clinical applications, we focused on stem cells for the analysis of cell-material interactions at the molecular level for further experiments.

Effects of Peptide Nanofibers with Different Morphologies on the Osteochondrogenic Differentiation of MSCs. When cells are exposed to a microenvironment, their morphology, spreading area, and proliferation rate are altered, resulting in the activation of specific response mechanisms that directly affect the organism at cell and tissue levels. ${ }^{45} \mathrm{~A}$ thorough understanding of cell-materials interactions is essential to regulate these responses. These effects are also important for the clinical applicability of stem cells, which are being utilized for the treatment of various diseases and biomedical applications. The primary advantage of stem cells is their ability to differentiate into multiple lineages, which can be mediated through their cultivation on peptide scaffolds or other biomaterials. Thus, the differentiation potential of rMSCs were analyzed on peptide nanofibers with different morphology and chirality. Mesenchyme-originated stem cells may differentiate into osteogenic, chondrogenic, and adipogenic lineages, and the initial commitment of cells to differentiate into the osteogenic and chondrogenic lineages was investigated by qRTPCR analysis on day 7. Runx-2, a transcriptional factor, which is expressed during the osteogenic differentiation of stem cells to activate the synthesis of bone specific proteins, was found to be upregulated on both L- and D-forms of cylindrical nanofibers (LVV and D-VV), with L-VV nanofibers exhibiting a more pronounced effect on Runx-2 expression than the D-form 
A

L-VV D-VV L-FF D-FF

Integrin $\beta 1$

GAPDH
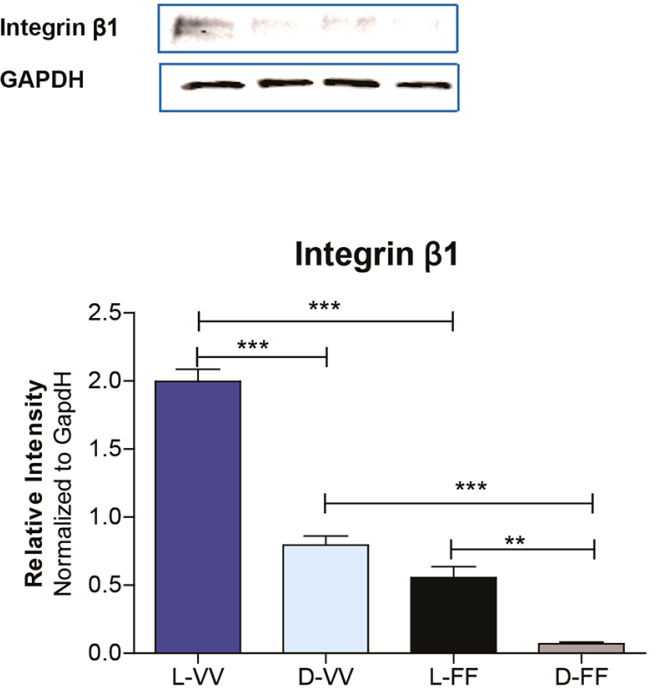

C
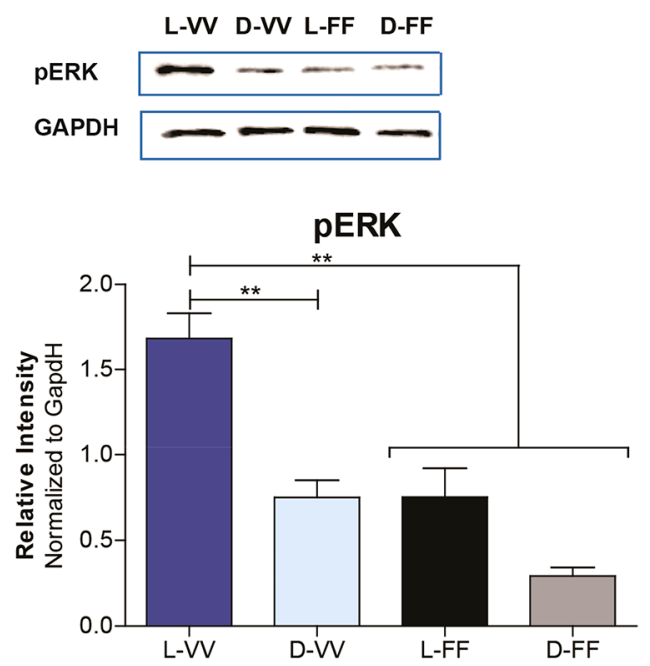

B

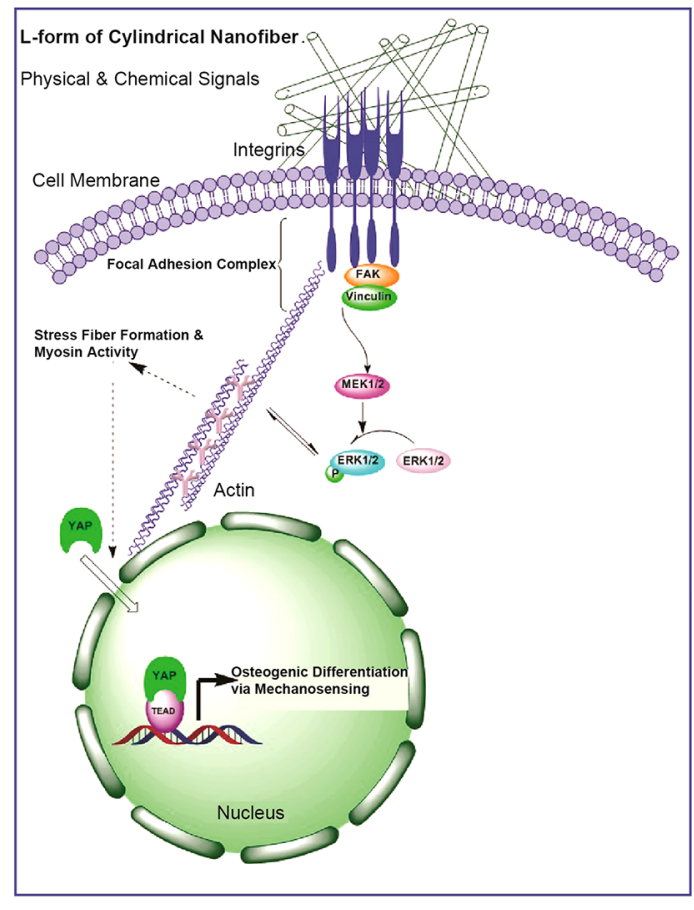

D

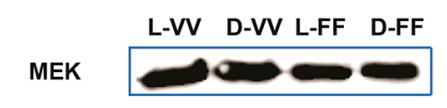

GAPDH

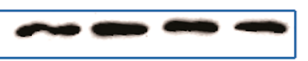

MEK

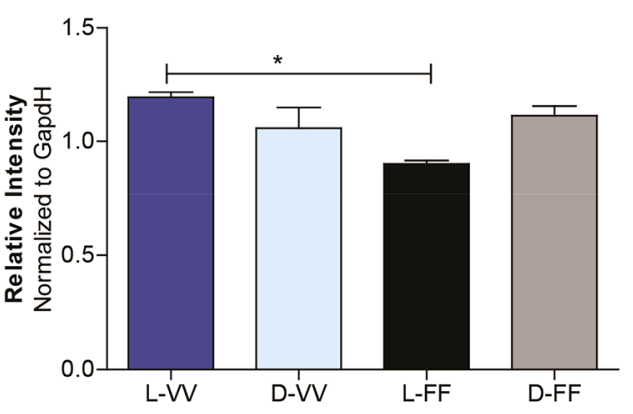

Figure 3. Signaling pathway analyses of cellular mechanotransduction. (A) Integrin $\beta 1$ expression on peptide nanofibers with different morphology and chirality, as analyzed by Western blotting $(* * p<0.01, * * * p<0.001$ by one-way ANOVA with Bonferroni's posthoc test, mean \pm s.e.m.). (B) Schematic representation of the signaling pathway activated in MSCs cultured on the L-form of cylindrical nanofibers. (C) pERK expression on peptide nanofibers, as analyzed by Western blotting $(* * p<0.01$ by one-way ANOVA with Bonferroni's posthoc test, mean \pm s.e.m.). (D) MEK expression on peptide nanofibers, as analyzed by Western blotting $(* p<0.05$ by one-way ANOVA with Bonferroni's posthoc test, mean \pm s.e.m.).

(Figure 2D). In contrast, twisted ribbon-like nanofibers (L-FF and D-FF) significantly inhibited osteogenic differentiation of MSCs. These twisted ribbon-like nanofibers (L-FF and D-FF) instead increased the chondrogenesis of stem cells, as shown by increased in Sox-9 expression, which was especially significant on D-FF nanofibers (Figures 2D and S18).

Scaffold-Mediated Structural and Chiral Regulation of Mechanotransduction. Since both twisted ribbon-like and cylindrical nanofiber systems presented the same amino acid sequences on their periphery, the differential bioactivity of these nanofibers was analyzed to investigate the effects of morphology and chirality on mechanotransduction of cells. Protein expression levels of key regulators for mechanotransduction-specific molecular pathways were investigated by
Western blot analysis to further evaluate the effect of cellmaterial interactions of rMSCs on peptide nanofiber scaffolds. Cellular mechanotransduction is initiated by the binding of stretch receptors to nanofibers, which activates a protein cascade to convert mechanical information into biochemical signals. ${ }^{45}$ Integrin $\beta 1$ receptor is a key player in the early steps of this process and forms a heterodimeric complex with several integrin $\alpha$ receptors to bind fibrous proteins of the ECM and initiate the formation of focal adhesion complexes. Western blotting analysis of integrin $\beta 1$ showed that its expression is upregulated on the L-forms of cylindrical $(\mathrm{L}-\mathrm{VV})$ and twisted ribbon-like nanofibers (L-FF) compared to the D-forms (Figure $3 \mathrm{~A})$. Between the two distinct morphologies, cylindrical nanofibers (VV-) significantly increased the expression of 


\section{Vinculin Actin Nucleus}
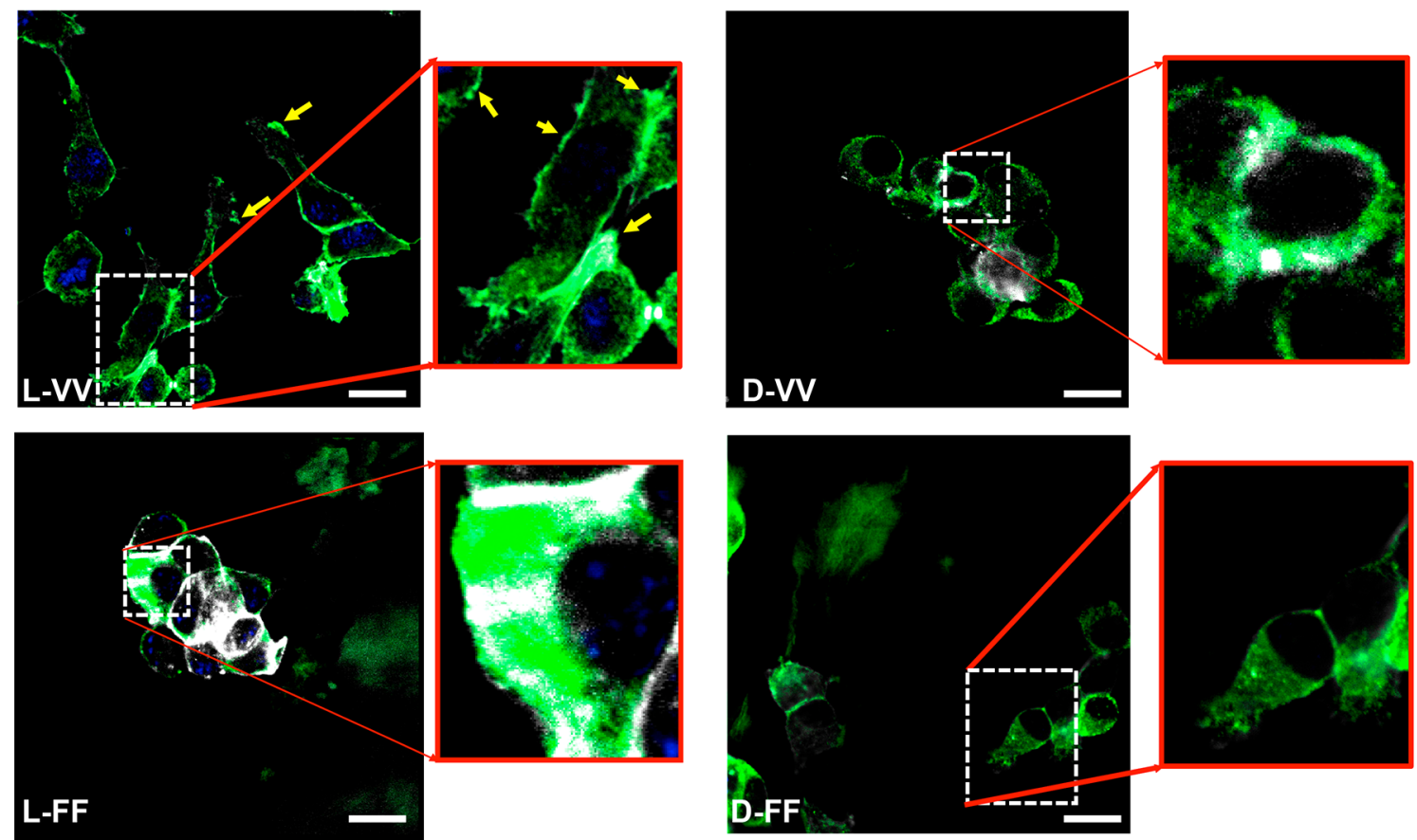

Figure 4. Focal adhesion complex analyses of MSCs on peptide nanofibers with different morphology and chirality; Confocal images of MSCs on peptide nanofibers stained for the visualization of actin stress fibers (gray), vinculin (green), and nuclei (blue), scale bars $=20 \mu \mathrm{m}$ ).

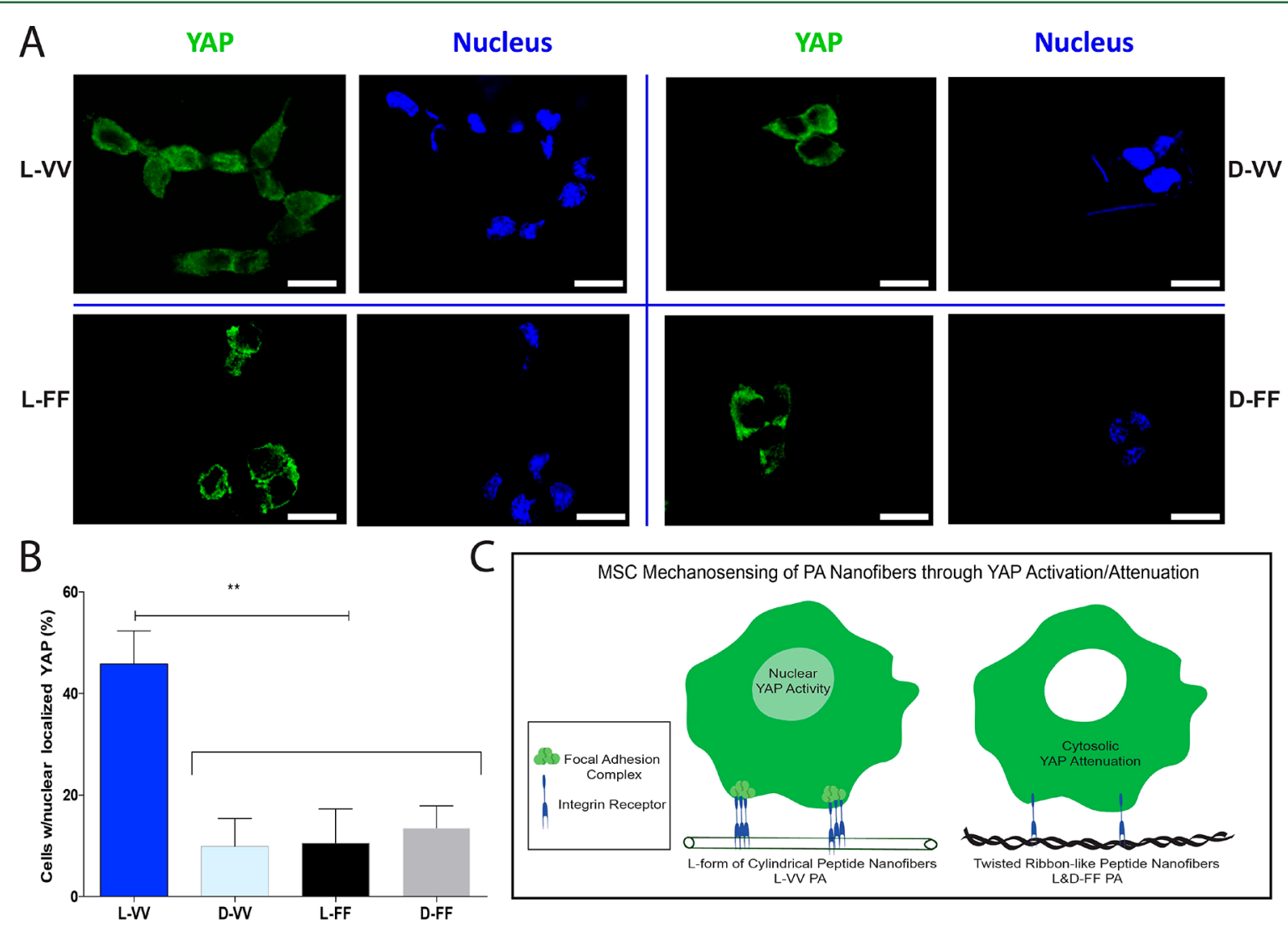

Figure 5. Cellular mechanosensing of peptide nanofibers with different morphology and chiralities through YAP activation/attenuation. (A) Confocal microscope images of MSCs stained for the visualization of YAP proteins (green) and nuclei (blue), scale bars $=20 \mu \mathrm{m}$. (B) Cells with nuclear YAP localization were quantified from the confocal images, which shown as percent of cells in the all counted cells $(* * p<0.01$ by one-way ANOVA with Bonferroni's posthoc test, mean \pm s.e.m.). (C) Schematic representation of MSC response on different PA nanofibers through activation/attenuation of YAP protein into the nucleus/cytosol. 
integrin $\beta 1$ compared to twisted ribbon-like nanofibers. In addition, $\mathrm{L}-\mathrm{VV}$ also had a greater capacity to induce integrin $\beta 1$ expression in MSCs compared to D-VV, suggesting the importance of chirality for cell-ECM interactions.

While integrin receptors provide the initial interaction of peptide nanofibers with cells, the transduction of this information entails a complex signaling cascade (Figure 3B). The phosphorylation of ERK and MEK is an integral step in this process and was investigated by Western blotting analysis. Significant differences were observed in the expression of MEK in MSCs on peptide nanofibers with different morphologies; in particular, rMSCs on L-form nanofibers invariably had higher MEK levels than their D-form counterparts, and cylindrical nanofibers (L-VV) had increased MEK expression compared to twisted ribbon (L-FF) nanofibers (Figure 3D). In addition, MSCs cultured on peptide nanofibers all expressed phosphorylated and activated ERK, but at different levels (Figure 3C; nonactivated ERK was not detected in the groups and is not shown). The pERK expression patterns were similar to integrin $\beta 1$, as $\mathrm{L}$-forms of both VV- and FF-nanofibers had enhanced pERK expression, and cylindrical nanofibers enhanced phosphorylated ERK levels to a greater extent than twisted ribbon-like nanofibers.

Vinculin expression was analyzed by both flow cytometry and confocal microscopy to determine the changes in focal adhesion point formation on peptide scaffolds (Figures 4 and S19B). The localization of vinculin proteins is essential for the analysis of focal adhesion complexes since vinculin plays an essential role in strengthening the adhesion complex by bearing force loads during the adhesion process. ${ }^{44,46}$ According to the confocal microscopy analysis, actin filaments were organized into well-defined stress fibers in cells on L-VV nanofibers (Figure S20). In addition, vinculin was strongly expressed on the protrusions of the cell membrane where focal adhesion complexes are formed. In contrast, on $\mathrm{D}-\mathrm{VV}$ nanofibers, cells did not form stress fibers, vinculin proteins were observed in the cytosol, and the cells were not observed to develop long filopodia. Similarly, on twisted ribbon-like nanofibers (L-FF and D-FF), cells failed to develop stress fibers or form cellular protrusions; instead, they retained a spherical morphology with few adhesion points.

To demonstrate the molecular effect of peptide nanofiber morphology and chirality on cellular processes, the expression of YAP (Yes-associated protein, which is a transcriptional coactivator involved in organ growth) was assayed by immunocytochemical analyses. YAP is a transcription coactivator that regulates many cellular processes by shuttling between the nucleus and cytosol and interacting with transcription factors to inhibit or activate the transcription process. ${ }^{47}$ The mechanical regulation of YAP activity involves the activation of F-actin capping/severing proteins and the formation of stress fibers, which function as a mechanical rheostat in the mechanotransduction of cells. ${ }^{47,48}$ When YAP proteins are active, they localize into the nucleus and function as transcriptional coactivators; however, when they are inactive, they remain in the cytosol (Figure 5B). According to confocal analyses, cells having nuclear YAP localization were quantified, and the number of cells that had YAP in their nuclei was found to be significantly higher in L-VV group compared to other groups, suggesting that the activation of YAP occurs within 24 $\mathrm{h}$ of cell seeding on L-VV nanofibers (Figure 5A). However, most of the MSCs cultured on the D-form of this cylindrical nanofiber (D-VV) had still inactive YAP proteins in their cytosol after $24 \mathrm{~h}$ incubation. Similarly, most of the cells on twisted ribbon-like nanofibers (both L-and D-from of FF- nanofibers) had inactive YAP proteins in their cytosols (Figure 5A,B). Thus, the influence of supramolecular chirality on cells is decisive on even their gene-level regulation and introduces an additional level of complexity to the structural and mechanical effects of nanofibers.

\section{DISCUSSION}

In addition to their successful use in medical applications, supramolecular nanostructures present a great opportunity to study cell-materials interactions by emulating the native ECM. ${ }^{13,15}$ Limitations in the in-depth analysis of cell-material interactions at the molecular level complicate potential investigations into the mechanical regulation of cells through morphological signals provided by the extracellular environment. Here we designed and synthesized a series of nanofiber scaffolds with well-defined structural properties, and used them to investigate the effects of fiber morphology and chirality on cellular behavior. Each peptide nanostructure was synthesized homogeneously and showed no variance in physical and chemical features. L-VV and D-VV peptide nanofibers exhibited cylindrical morphology while L-FF and D-FF peptide nanofibers had a twisted ribbon-like morphology, as characterized by TEM analyses. Their chiral signatures were further analyzed and confirmed by CD measurements.

Peptides and proteins regulate their chirality through the selfassembly of their amino acid residues. ${ }^{49}$ Therefore, different supramolecular systems with distinct structural features can be developed by altering assembly dynamics through the use of $\mathrm{L}$ or D-enantiomers of amino acids. ${ }^{50}$ In addition, self-assembly of nanomaterials can be reconfigured, and unique mechanical properties and complex topologies can be developed by controlling the interfacial tension of chiral materials. ${ }^{51}$ Here we showed $\mathrm{L}^{-}$and $\mathrm{D}$-forms of morphologically different nanofibers and observed that differences in chirality and morphology can result in the activation or repression of distinctive cellular processes. In rheology measurements, all peptide nanofiber networks displayed elastic solid-like behavior with high water content, and we did not detect any difference in elastic moduli of nanofibers between L- and D-forms. On the other hand, significant differences were observed in the elastic moduli of the $\mathrm{L}$ - and $\mathrm{D}$-forms of peptide nanofibers through the AFM measurement of nanofibers and nanobundles (Figures S13 and S14). All nanofibers have megapascal-level elastic modulus values in aqueous environment, which emulates the conditions experienced by cells. Although the effect of stiffness on cellular behavior is well-documented in the literature, such studies generally focus on the mechanical properties of bulk gels. ${ }^{52}$ In this study, AFM force map analysis provided deep insights for the elastic behavior of nanofibers, which were not detected by rheological analysis. When considering the concentration difference between two measurements, rheology provided bulk macro-scale measurement depending on the highly concentrated nanofiber network properties where molecular interactions could not be detected precisely. On the other hand, AFM provided nanoscale mechanical analyses, which is the scale at which cellular interactions occur. In addition, it was previously indicated that rather than bulk stiffness, mechanical feedback gathered from the interaction through collagen fibrils and integrin receptors has profound effect on the stem cell behavior. ${ }^{53}$ Similarly, we found that both morphology and chirality of nanofibers had profound effects on 
their mechanical stiffness: L-VV nanofibers formed more stiff fibrils than D-VV and FF-peptide nanofibers, which is relevant to their biological activity that we observed due to the wellknown impact of stiffness on the initiation of osteochondral differentiation. ${ }^{54,55}$ As observed in the results of molecular dynamics simulations, D-VV had lower hydrogen bonding density than the L-form due to side-chain steric hindrance, which affects the rigidity of nanofiber formation. Similarly, twisted ribbon-like nanofibers had lower hydrogen bonding density according to molecular dynamics simulations, and the red shift in the $\mathrm{CD}$ spectrum of FF containing nanofibers is indicative of weaker hydrogen bond formation. $\mathrm{L}-\mathrm{VV}$, on the other hand, has a much higher H-bond density, which allows the rigid packing of structure and results in stiffer nanofiber formation, while lower H-bond density and weaker bond formation cause looser packing of PAs during self-assembly of nanofibers. The crucial role of rigid backbone in viscoelastic properties was also shown in a previous study. ${ }^{56}$ Hence, the nanofibers with different morphology and chirality were developed through strictly controlled modifications in the design of peptide amphiphile molecules.

In this study, stem cells exhibited differential responses to different morphological and chiral signals. Osteogenesis and cell spreading were strongly stimulated on the L-form of cylindrical nanofibers (L-VV) compared to D-VV, L-FF, and D-FF groups. This result is consistent with the previous reports, as a high spread area has been reported to promote the osteogenic differentiation of MSCs. ${ }^{57}$ The spreading of cells provides them with higher contractility through increased stress fiber formation, which increases their response to soluble factors such as autocrine/paracrine Wnt signals, as well as osteogenic media supplements. ${ }^{58}$ In addition, Sox-9 expression was also detected in FF-groups. Sox-9 is indeed expressed during both osteogenic and chondrogenic differentiation at the earlier phase of mesenchymal differentiation; consequently, Sox-9 expression in the presence of osteogenic markers (e.g., Runx-2) is considered to be an indicator of osteogenesis, while Sox-9 in the absence of osteogenic gene expression is a marker of chondrogenesis. Indeed, in the study of Akiyama et al., osteochondroprogenitor cells, as well as progenitors in a variety of tissues, were shown to be derived from Sox-9-expressing precursors during mouse embryogenesis, and inactivation of Sox- 9 resulted in prevention of mature osteoblast formation. ${ }^{59}$ Hence, we also showed both L- and D-form of cylindrical nanofibers increased the Sox- 9 expression at mRNA levelwhere L-VV had more pronounced effect. On the other hand, twisted ribbon-like nanofibers increased the Sox-9 expression in the absence of Runx-2 and promoted the chondrogenesis of MSCs. In fact, both L-and D-forms of the twisted ribbon nanofibers greatly reduced cellular proliferation as well as cellular spreading, which resulted in substantially lower osteogenesis. This restriction of cellular spreading was also seen for HUVECs and hFib cells cultured on the L-form of twisted ribbon-like nanofibers. Interestingly, the morphological effect on cellular spreading was more profoundly seen on the Lform of twisted ribbon-like nanofibers, which also indicates the importance of chiral signatures for the regulation of cellular behavior. For cellular adhesion, differences among nanofiber groups were only observed for HUVECs, which is probably due to this cell line's inherent susceptibility toward physical changes in environment: endothelial cells are required to stretch, withstand strong shear forces, and prevent the development of atherosclerotic plaques and aberrant neovascularization in their native environment. ${ }^{60}$ Therefore, the difference in the morphology of the nanofibers had a profound effect on the adhesion of HUVECs.

The minimal cellular proliferation and the spherical, nonadhering morphology of rMSCs on FF-PAs were conducive for chondrogenic differentiation, which was further enhanced when the cells were exposed to chondrogenic medium (Figure S18). The regulation of cell spreading is an essential requirement for optimal cellular differentiation, and high cell density have been reported to stimulate the chondrogenic differentiation of MSCs; ${ }^{57}$ however, we observed scaffolddriven chondrogenesis at a relatively lower cellular density on D-FF nanofibers. The combination of structural and morphological signals from the nanofiber surface and soluble chondrogenic factors from the differentiation medium further enhanced the chondrogenesis process on the D-form of ribbonlike nanofibers. Interestingly, while ribbon-like nanofibers significantly inhibited osteogenesis, they also strongly promoted chondrogenesis, especially in their D-form. As such, peptide scaffold morphology can be used to control the differentiation of MSCs into two closely related lineages. The spreading behavior of rMSCs on VV- and FF-nanofibers is also consistent with previous research, ${ }^{57,61}$ as the less-spread cells on ribbonlike nanofibers differentiated into chondrogenic lineage, while the well-spread cells on cylindrical nanofibers committed to osteogenesis.

To better understand the underlying cellular mechanisms of these distinct responses, we further analyzed the specific components of the signaling pathways that are associated with the cellular adhesion, migration, and differentiation of MSCs. Kilian et al. previously showed that cell contractility, which is directly related to spreading of cells, activates the ERK/JUN pathway. ${ }^{58}$ We also observed an increase in p-ERK expression in MSCs on L-VV nanofibers and a significantly higher potential for osteogenesis through the activation of ERK/MAPK pathways even on the first day of culture. ${ }^{62,63}$ In addition, in the MEK1-2 (MAP/ERK kinase 1-2) expressions of cells on L-VV nanofibers were significantly increased. ERK pathway activation is also strongly related with the increase of integrin $\beta 1$ expression, and we found that both proteins were highly expressed in cells on L-VV nanofibers. Integrins interact with ECM fibrils and intracellular actin filaments through cytosolic linker proteins, which facilitate the mechanical connection of intracellular and extracellular environment of cells to raise specific cellular responses. ${ }^{44}$ In addition, integrins act as transducers in the cellular sensing of physical forces that are exerted through the surrounding environment of cells. ${ }^{44,45}$ The $\mathrm{L}$-form of cylindrical nanofibers increased integrin $\beta 1$ expression and promoted the activation of ERK pathway, which stimulated the osteogenic differentiation of MSCs. This activation was also linked to the activation of F-actin, leading to the formation of well-organized stress fibers that were observed by confocal microscopy through actin staining. Interestingly, this effect of cylindrical nanofibers was much more advanced in the L-form compared to the D-form, which underlines the importance of the chiral signature of supramolecular nanofiber networks for the recognition by integrin receptors and the activation of signaling pathways.

For the analysis of focal adhesion complexes, vinculin expression was evaluated by using both confocal microscopy and flow cytometry (Figure S19B). Vinculin is responsible for bearing the forces exerted on the focal adhesion complex, and its presence is essential for anchoring the complex to the 
surrounding actin network. ${ }^{44,46}$ According to confocal microscopy analyses, culturing on L-VV nanofibers significantly increased focal adhesion complex formation on the lamellipodial membranes of cells compared to other groups. In addition, flow cytometry results revealed a marked increase in the expression of vinculin in cells cultured on D-VV nanofibers, which may have enhanced the formation of focal adhesion complexes at later days of culture; albeit not as quickly as L-VV nanofibers. Interestingly, we also observed a similar increase in the expression of Integrin $\alpha 2$, which forms a heterodimer complex with Integrin $\beta 1$ and has been shown to bind to collagen and fibrinogen fibrils in $\mathrm{ECM}^{45}$ (Figure S19A). This result further supported the hypothesis that $\mathrm{D}-\mathrm{VV}$ nanofibers increased the expression of fibrillar protein-binding integrins, which can be later recruited for the formation of focal adhesion complexes, but not as quickly as on $\mathrm{L}-\mathrm{VV}$ nanofibers. In addition, an increase in FAK protein expression was observed in most of the cells on D-VV nanofibers, at a level that was significantly higher than other peptide nanofibers (Figure S19C). Overall, these findings all indicate that culturing on DVV nanofibers caused a delayed response in the formation of focal adhesion complexes when compared to L-VV nanofibers. While the spreading areas of $\mathrm{L}-\mathrm{VV}$ and $\mathrm{D}-\mathrm{VV}$ groups are similar, long-term adhesive forces have been shown to influence cellular behavior to a greater extent than spreading, suggesting that mechanotransduction may be more prominent on L-VV (as evidenced by higher YAP nuclearization and osteogenic differentiation) despite comparable cell areas. In addition, while vinculin and FAK expression were higher on $\mathrm{D}-\mathrm{VV}$ nanofibers, vinculin in this group was confined to the cytoplasm and the number of focal adhesions was less compared to L-VV (Figures S19 and 4). Consequently, we believe that D-VV shows an effect that is similar to but less pronounced than L$\mathrm{VV}$, such that the cells are able to spread but do not establish strong connections with the substrate. The higher expressions of integrin alpha-2, FAK, and vinculin may be a result of the fact that cells on $\mathrm{D}-\mathrm{VV}$ are at a stage where focal adhesion complexes are in the process of being established, while the mature connections formed on $\mathrm{L}-\mathrm{VV}$ are maintained by comparatively lower expressions of focal adhesion point-related proteins. ${ }^{64}$ As a functional demonstration of this effect, cells spread more on $\mathrm{D}-\mathrm{VV}$ after 3 days of incubation than 1 day incubation (Figure S16).

The twisted ribbon-like nanofibers limited the spreading of MSCs, decreased focal adhesion points and actin fibers, and deactivated the ERK pathway through diminished Integrin $\beta 1$ expression. Matrix-induced cell rounding has previously been reported to correspond directly to decreases in focal adhesion and ERK pathway activation, ${ }^{53}$ which is caused by alterations in the mechanical feedback that occurs between collagen tethers and anchoring proteins on the cell membrane. As such, the suppression of integrin ligation in the twisted ribbon-like nanofibers appears to limit the adhesion of rMSCs resulting in insufficient mechanical feedback and limited ERK/MAPK pathway signaling. Although osteogenesis was not observed on FF containing nanofibers, chondrogenesis was significantly promoted on these scaffolds (and especially on the D-FF form), which is an interesting result that warrants further characterization and may be utilized for the development of smart biomaterial platforms for cartilage tissue engineering.

The effect of mechanical cues from the extracellular environment was also reflected on the gene expression profiles of rMSCs for the previously mentioned cellular signaling pathways. We observed that the cytosolic/nuclear localization of YAP protein was altered in cells grown on supramolecular peptide nanofibers. YAP/TAZ is a pair of transcriptional coactivators that play critical roles in organ growth and have been shown to be essential in the mechanotransduction of cells through their activity as nuclear sensors of stiffness. ${ }^{47,65}$ In particular, extracellular mechanical signals resulting from substrate effects or excessive cell growth trigger the organization of F-actin and enhance the nuclear localization of YAP. ${ }^{65}$ Similarly, we found that MSCs on L-form cylindrical nanofibers (L-VV) had stronger integrin $\beta 1$ expression, activated ERK signaling, and nuclear YAP activity compared to other groups. In contrast, cells on twisted ribbon-like nanofibers had low proliferation, confined cellular spreading and less focal adhesion, which resulted in the cytoplasmic retention of YAP. Even though the D-form of cylindrical nanofibers (D-VV) had a similar bulk morphology to the Lform, cells on the L-form had higher nuclear YAP activity compared to the $\mathrm{D}$-form, suggesting that different chiral signals result in different routes of mechanotransduction in cells. The correlation between the decrease in proliferation and YAP activity also agrees with recent studies on the regulation of YAP/TAZ nuclear activity and proliferation by $\mathrm{N}$-cadherin and RGD-incorporated hydrogels. ${ }^{48}$

\section{CONCLUSION}

In summary, we showed that mechanosensitive pathways are selectively activated in stem cells depending on the morphology and chirality of the peptide nanofibers in the microenvironment. The mechanosensation and transduction controlled the activity of the YAP/TAZ complex, which relayed the effects of the microenvironment to inside of the cells through integrin $\beta 1$ receptor binding and subsequent cytosolic protein activations in the ERK pathway, and ultimately result in the nuclear localization of YAP in response to L-amino acid containing cylindrical nanofibers. However, this response was modulated not only by mechanical signaling, but also by the chemical signatures of the nanofibers and their chiral features. Morphology of the nanofibers was primarily responsible for initiating the cellular responses but had synergistic effects with chiral signals. L-Enantiomers found in natural proteins were observed to exhibit an increased effect on cell responses when introduced on cylindrical nanofibers, enhancing their capacity for promoting osteogenic differentiation even without incorporation of a bioactive epitope. D-Enantiomers, on the other hand, had a pronounced effect on chondrogenesis when introduced in ribbon-like nanofibers. Overall, these results show that the morphology and chirality of the nanofibers can be exploited for selective differentiation of stem cells for regenerative medicine applications. In addition, it is interesting to note that fundamental differences in hydrogen bond formation between peptide enantiomers may have played an important role during the early evolution of proteins, potentially accounting for the fundamental chiral asymmetry that exists in all known life.

\section{ASSOCIATED CONTENT}

\section{S Supporting Information}

The Supporting Information is available free of charge on the ACS Publications website at DOI: 10.1021/acs.biomac.7b00773. 
Chemical structures of PAs; primer sequences for qRTPCR experiments; peptide nanofiber compositions; LCMS results; TEM images of PA nanofibers; SAXS analyses; molecular dynamics simulations; rheological characterizations of nanofibers; AFM characterizations; Alamar Blue, Live/Dead, and adhesion assay results for rMSCs; additional spreading results for HUVECs and hFibs; chondrogenic differentiation analyses of rMSCs; protein expression analyses of Integrin $\alpha 2$ and FAK by Western blotting; protein expression analyses of Vinculin by flow cytometry analyses; confocal microscopy images of actin filaments of rMSCs (PDF)

\section{AUTHOR INFORMATION}

\section{Corresponding Authors}

*E-mail: atekinay@bilkent.edu.tr.

*E-mail: mguler@uchicago.edu.

\section{ORCID ${ }^{\circ}$}

Valeria Castelletto: 0000-0002-3705-0162

Ian Hamley: 0000-0002-4549-0926

Ayse B. Tekinay: 0000-0002-4453-814X

Mustafa O. Guler: 0000-0003-1168-202X

\section{Author Contributions}

In vitro cell culture studies were designed and performed by E.A., O.U., and A.B.T.; chemical synthesis and characterizations were designed and performed by M.H.K., E.A., R.G., and M.O.G.; AFM measurements and analyses were performed by B.D., A.E.T., E.A., A.D.O., and A.D.; molecular dynamics simulations were performed by S.B.; SAXS analyses were performed by D.H.M., V.C., C.E.G., and I.H. The manuscript was written by E.A., A.B.T., and M.O.G. with contributions of all authors. All authors have given approval to the final version of the manuscript.

\section{Notes}

The authors declare no competing financial interest.

\section{ACKNOWLEDGMENTS}

E.A. acknowledges support from TUBITAK-BIDEB fellowship. R.G. was partially supported by RFBR (Grant No. 16-3360146) and Program of Competitive Growth of Kazan Federal University. This work is partially supported by TUBITAK 114Z728, TUBA, and Marie Curie International Reintegration Grants. I.W.H. thanks EPSRC (U.K.) for the award of a Platform Grant (ref EP/L020599/1). We would like to express our gratitude to Dr. B. Senturk for valuable comments on the manuscript and M. Guler for assistance on TEM. We thank Diamond Light Source and the ESRF for beam time.

\section{ABBREVIATIONS}

PA, peptide amphiphile; ECM, extracellular matrix; FBS, fetal bovine serum; CD, circular dichroism; TEM, transmission electron microscopy; SEM, scanning electron microscopy; qRT-PCR, quantitative real time polymerase chain reaction; prep-HPLC, preparative high performance liquid chromatography; s.e.m., standard error of mean

\section{REFERENCES}

(1) Guilak, F.; Cohen, D. M.; Estes, B. T.; Gimble, J. M.; Liedtke, W.; Chen, C. S. Control of stem cell fate by physical interactions with the extracellular matrix. Cell stem cell 2009, 5 (1), 17-26.
(2) Koide, T. Designed triple-helical peptides as tools for collagen biochemistry and matrix engineering. Philos. Trans. R. Soc., B 2007, 362 (1484), 1281-1291.

(3) Knight, C. G.; Morton, L. F.; Peachey, A. R.; Tuckwell, D. S.; Farndale, R. W.; Barnes, M. J. The Collagen-binding A-domains of Integrins $\alpha 1 \beta 1$ and $\alpha 2 \beta 1$ Recognize the Same Specific Amino Acid Sequence, GFOGER, in Native (Triple-helical) Collagens. J. Biol. Chem. 2000, 275 (1), 35-40.

(4) Li, B.; Moshfegh, C.; Lin, Z.; Albuschies, J.; Vogel, V. Mesenchymal stem cells exploit extracellular matrix as mechanotransducer. Sci. Rep. 2013, 3, 2425.

(5) Muiznieks, L. D.; Keeley, F. W. Molecular assembly and mechanical properties of the extracellular matrix: A fibrous protein perspective. Biochim. Biophys. Acta, Mol. Basis Dis. 2013, 1832 (7), $866-875$.

(6) Das, R. K.; Zouani, O. F.; Labrugère, C.; Oda, R.; Durrieu, M.-C. Influence of Nanohelical Shape and Periodicity on Stem Cell Fate. ACS Nano 2013, 7 (4), 3351-3361.

(7) Buxboim, A.; Ivanovska, I. L.; Discher, D. E. Matrix elasticity, cytoskeletal forces and physics of the nucleus: how deeply do cells 'feel'outside and in? J. Cell Sci. 2010, 123 (3), 297-308.

(8) Liu, G. F.; Zhang, D.; Feng, C. L. Control of Three-Dimensional Cell Adhesion by the Chirality of Nanofibers in Hydrogels. Angew. Chem., Int. Ed. 2014, 53 (30), 7789-7793.

(9) Sun, T.; Han, D.; Rhemann, K.; Chi, L.; Fuchs, H. Stereospecific interaction between immune cells and chiral surfaces. J. Am. Chem. Soc. 2007, 129 (6), 1496-1497.

(10) Zhang, M.; Qing, G.; Sun, T. Chiral biointerface materials. Chem. Soc. Rev. 2012, 41 (5), 1972-1984.

(11) Jakschitz, T. A.; Rode, B. M. Chemical evolution from simple inorganic compounds to chiral peptides. Chem. Soc. Rev. 2012, 41 (16), 5484-5489.

(12) Krause, E.; Bienert, M.; Schmieder, P.; Wenschuh, H. The helixdestabilizing propensity scale of D-amino acids: the influence of side chain steric effects. J. Am. Chem. Soc. 2000, 122 (20), 4865-4870.

(13) Arslan, E.; Garip, I. C.; Gulseren, G.; Tekinay, A. B.; Guler, M. O. Bioactive Supramolecular Peptide Nanofibers for Regenerative Medicine. Adv. Healthcare Mater. 2014, 3 (9), 1357-1376.

(14) Barker, T. H. The role of ECM proteins and protein fragments in guiding cell behavior in regenerative medicine. Biomaterials 2011, 32 (18), 4211-4214.

(15) Webber, M. J.; Kessler, J.; Stupp, S. I. Emerging peptide nanomedicine to regenerate tissues and organs. J. Intern. Med. 2010, 267 (1), 71-88.

(16) Tansik, G.; Kilic, E.; Beter, M.; Demiralp, B.; Sendur, G. K.; Can, N.; Ozkan, H.; Ergul, E.; Guler, M. O.; Tekinay, A. B. A glycosaminoglycan mimetic peptide nanofiber gel as an osteoinductive scaffold. Biomater. Sci. 2016, 4 (9), 1328-1339.

(17) Ceylan, H.; Kocabey, S.; Tekinay, A. B.; Guler, M. O. Surfaceadhesive and osteogenic self-assembled peptide nanofibers for bioinspired functionalization of titanium surfaces. Soft Matter 2012, 8 (14), 3929-3937.

(18) Gulseren, G.; Yasa, I. C.; Ustahuseyin, O.; Tekin, E. D.; Tekinay, A. B.; Guler, M. O. Alkaline phosphatase-mimicking peptide nanofibers for osteogenic differentiation. Biomacromolecules 2015, 16 (7), 2198-2208.

(19) Senturk, B.; Cubuk, M. O.; Ozmen, M. C.; Aydin, B.; Guler, M. O.; Tekinay, A. B. Inhibition of VEGF mediated corneal neovascularization by anti-angiogenic peptide nanofibers. Biomaterials 2016, 107, 124-132.

(20) Yasa, I. C.; Gunduz, N.; Kilinc, M.; Guler, M. O.; Tekinay, A. B. Basal Lamina Mimetic Nanofibrous Peptide Networks for Skeletal Myogenesis. Sci. Rep. 2015, 5, 16460-16460.

(21) Markvoort, A. J.; Ten Eikelder, H. M.; Hilbers, P. A.; de Greef, T. F.; Meijer, E. Theoretical models of nonlinear effects in twocomponent cooperative supramolecular copolymerizations. Nat. Commun. 2011, 2, 509.

(22) Zhang, L.; Wang, X.; Wang, T.; Liu, M. Supramolecular Gels: Tuning Soft Nanostructures in Self-assembled Supramolecular Gels: 
From Morphology Control to Morphology-Dependent Functions (Small 9-10/2015). Small 2015, 11 (9-10), 1024-1024.

(23) Chen, G.; Ushida, T.; Tateishi, T. Development of biodegradable porous scaffolds for tissue engineering. Mater. Sci. Eng., C 2001, 17 (1), 63-69.

(24) Green, D. W.; Lee, J.-M.; Kim, E.-J.; Lee, D.-J.; Jung, H.-S. Chiral Biomaterials: From Molecular Design to Regenerative Medicine. Adv. Mater. Interfaces 2016, 3 (6), 1500411.

(25) Breßler, I.; Kohlbrecher, J.; Thünemann, A. F. SASfit: a tool for small-angle scattering data analysis using a library of analytical expressions. J. Appl. Crystallogr. 2015, 48 (5), 1587-1598.

(26) Castelletto, V.; Cheng, G.; Stain, C.; Connon, C.; Hamley, I. Self-assembly of a peptide amphiphile containing L-carnosine and its mixtures with a multilamellar vesicle forming lipid. Langmuir 2012, 28 (31), 11599-11608.

(27) Castelletto, V.; Gouveia, R. M.; Connon, C. J.; Hamley, I. W. New RGD-peptide amphiphile mixtures containing a negatively charged diluent. Faraday Discuss. 2013, 166, 381-397.

(28) Pabst, G.; Rappolt, M.; Amenitsch, H.; Laggner, P. Structural information from multilamellar liposomes at full hydration: full qrange fitting with high quality x-ray data. Phys. Rev. E: Stat. Phys., Plasmas, Fluids, Relat. Interdiscip. Top. 2000, 62 (3), 4000.

(29) Tekin, E. D. Molecular dynamics simulations of self-assembled peptide amphiphile based cylindrical nanofibers. RSC Adv. 2015, 5 (82), 66582-66590.

(30) Phillips, J. C.; Braun, R.; Wang, W.; Gumbart, J.; Tajkhorshid, E.; Villa, E.; Chipot, C.; Skeel, R. D.; Kale, L.; Schulten, K. Scalable molecular dynamics with NAMD. J. Comput. Chem. 2005, 26 (16), $1781-1802$.

(31) MacKerell, A. D., Jr; Bashford, D.; Bellott, M.; Dunbrack, R. L., Jr; Evanseck, J. D.; Field, M. J.; Fischer, S.; Gao, J.; Guo, H.; Ha, S.; et al. All-atom empirical potential for molecular modeling and dynamics studies of proteins. J. Phys. Chem. B 1998, 102 (18), $3586-3616$.

(32) Essmann, U.; Perera, L.; Berkowitz, M. L.; Darden, T.; Lee, H.; Pedersen, L. G. A smooth particle mesh Ewald method. J. Chem. Phys. 1995, 103 (19), 8577-8593.

(33) Roe, D. R.; Cheatham, T. E., III PTRAJ and CPPTRAJ: software for processing and analysis of molecular dynamics trajectory data. $J$. Chem. Theory Comput. 2013, 9 (7), 3084-3095.

(34) Humphrey, W.; Dalke, A.; Schulten, K. VMD: visual molecular dynamics. J. Mol. Graphics 1996, 14 (1), 33-38.

(35) Niece, K. L.; Hartgerink, J. D.; Donners, J. J.; Stupp, S. I. Selfassembly combining two bioactive peptide-amphiphile molecules into nanofibers by electrostatic attraction. J. Am. Chem. Soc. 2003, 125 (24), 7146-7147.

(36) Pashuck, E. T.; Cui, H.; Stupp, S. I. Tuning supramolecular rigidity of peptide fibers through molecular structure. J. Am. Chem. Soc. 2010, 132 (17), 6041-6046.

(37) Arslan, E.; Guler, M. O.; Tekinay, A. B. Glycosaminoglycanmimetic signals direct the osteo/chondrogenic differentiation of mesenchymal stem cells in a three-dimensional peptide nanofiber extracellular matrix mimetic environment. Biomacromolecules 2016, 17 (4), 1280-1291.

(38) Pashuck, E. T.; Stupp, S. I. Direct observation of morphological tranformation from twisted ribbons into helical ribbons. J. Am. Chem. Soc. 2010, 132 (26), 8819-8821.

(39) Garifullin, R.; Guler, M. O. Supramolecular chirality in selfassembled peptide amphiphile nanostructures. Chem. Commun. 2015, 51 (62), 12470-12473.

(40) Micsonai, A.; Wien, F.; Kernya, L.; Lee, Y.-H.; Goto, Y.; Réfrégiers, M.; Kardos, J. Accurate secondary structure prediction and fold recognition for circular dichroism spectroscopy. Proc. Natl. Acad. Sci. U. S. A. 2015, 112 (24), E3095-E3103.

(41) Adler, A. J.; Greenfield, N. J.; Fasman, G. D. [27] Circular dichroism and optical rotatory dispersion of proteins and polypeptides. Methods Enzymol. 1973, 27, 675-735.
(42) Hovmöller, S.; Zhou, T.; Ohlson, T. Conformations of amino acids in proteins. Acta Crystallogr., Sect. D: Biol. Crystallogr. 2002, 58 (5), 768-776.

(43) Park, Y. B.; Kim, Y. Y.; Oh, S. K.; Chung, S. G.; Ku, S.-Y.; Kim, S. H.; Choi, Y. M.; Moon, S. Y. Alterations of proliferative and differentiation potentials of human embryonic stem cells during longterm culture. Exp. Mol. Med. 2008, 40 (1), 98-108.

(44) Schwartz, M. A. Integrins and extracellular matrix in mechanotransduction. Cold Spring Harbor Perspect. Biol. 2010, 2 (12), a005066.

(45) Prowse, A. B. J.; Chong, F.; Gray, P. P.; Munro, T. P. Stem cell integrins: Implications for ex-vivo culture and cellular therapies. Stem Cell Res. 2011, 6 (1), 1-12.

(46) Chanet, S.; Martin, A. C. Mechanical force sensing in tissues. Prog. Mol. Biol. Transl. Sci. 2014, 126, 317.

(47) Aragona, M.; Panciera, T.; Manfrin, A.; Giulitti, S.; Michielin, F.; Elvassore, N.; Dupont, S.; Piccolo, S. A mechanical checkpoint controls multicellular growth through YAP/TAZ regulation by actinprocessing factors. Cell 2013, 154 (5), 1047-1059.

(48) Cosgrove, B. D.; Mui, K. L.; Driscoll, T. P.; Caliari, S. R.; Mehta, K. D.; Assoian, R. K.; Burdick, J. A.; Mauck, R. L. N-cadherin adhesive interactions modulate matrix mechanosensing and fate commitment of mesenchymal stem cells. Nat. Mater. 2016, 15, 1297.

(49) Luo, Z.; Zhang, S. Designer nanomaterials using chiral selfassembling peptide systems and their emerging benefit for society. Chem. Soc. Rev. 2012, 41 (13), 4736-4754.

(50) Aggeli, A.; Nyrkova, I. A.; Bell, M.; Harding, R.; Carrick, L.; McLeish, T. C.; Semenov, A. N.; Boden, N. Hierarchical self-assembly of chiral rod-like molecules as a model for peptide $\beta$-sheet tapes, ribbons, fibrils, and fibers. Proc. Natl. Acad. Sci. U. S. A. 2001, 98 (21), 11857-11862.

(51) Gibaud, T.; Barry, E.; Zakhary, M. J.; Henglin, M.; Ward, A.; Yang, Y.; Berciu, C.; Oldenbourg, R.; Hagan, M. F.; Nicastro, D.; et al. Reconfigurable self-assembly through chiral control of interfacial tension. Nature 2012, 481 (7381), 348-351.

(52) Engler, A. J.; Sen, S.; Sweeney, H. L.; Discher, D. E. Matrix elasticity directs stem cell lineage specification. Cell 2006, 126 (4), 677-689.

(53) Trappmann, B.; Gautrot, J. E.; Connelly, J. T.; Strange, D. G.; Li, Y.; Oyen, M. L.; Stuart, M. A. C.; Boehm, H.; Li, B.; Vogel, V.; et al. Extracellular-matrix tethering regulates stem-cell fate. Nat. Mater. 2012, 11 (7), 642-649.

(54) Park, J. S.; Chu, J. S.; Tsou, A. D.; Diop, R.; Tang, Z.; Wang, A.; $\mathrm{Li}, \mathrm{S}$. The effect of matrix stiffness on the differentiation of mesenchymal stem cells in response to TGF- $\beta$. Biomaterials 2011, 32 (16), 3921-3930.

(55) Evans, N. D.; Minelli, C.; Gentleman, E.; LaPointe, V.; Patankar, S. N.; Kallivretaki, M.; Chen, X.; Roberts, C. J.; Stevens, M. M. Substrate stiffness affects early differentiation events in embryonic stem cells. Eur. cell mater. 2009, 18 (1), e13.

(56) Mondal, S.; Varenik, M.; Bloch, D. N.; Atsmon-Raz, Y.; Jacoby, G.; Adler-Abramovich, L.; Shimon, L. J.; Beck, R.; Miller, Y.; Regev, O. A minimal length rigid helical peptide motif allows rational design of modular surfactants. Nat. Commun. 2017, 8, 14018.

(57) Eyckmans, J.; Lin, G. L.; Chen, C. S. Adhesive and mechanical regulation of mesenchymal stem cell differentiation in human bone marrow and periosteum-derived progenitor cells. Biol. Open 2012, 1, $\mathrm{BIO} 20122162$.

(58) Kilian, K. A.; Bugarija, B.; Lahn, B. T.; Mrksich, M. Geometric cues for directing the differentiation of mesenchymal stem cells. Proc. Natl. Acad. Sci. U. S. A. 2010, 107 (11), 4872-4877.

(59) Akiyama, H.; Kim, J.-E.; Nakashima, K.; Balmes, G.; Iwai, N.; Deng, J. M.; Zhang, Z.; Martin, J. F.; Behringer, R. R.; Nakamura, T.; et al. Osteo-chondroprogenitor cells are derived from Sox9 expressing precursors. Proc. Natl. Acad. Sci. U. S. A. 2005, 102 (41), 1466514670.

(60) Park, H.-J.; Zhang, Y.; Georgescu, S. P.; Johnson, K. L.; Kong, D.; Galper, J. B. Human umbilical vein endothelial cells and human dermal microvascular endothelial cells offer new insights into the 
relationship between lipid metabolism and angiogenesis. Stem Cell Rev. Rep. 2006, 2 (2), 93-101.

(61) Gao, L.; McBeath, R.; Chen, C. S. Stem Cell Shape Regulates a Chondrogenic versus Myogenic Fate through Racl and N-cadherin. Stem Cells (Durham, NC, U. S.) 2010, 28 (3), 564-572.

(62) Jaiswal, R. K.; Jaiswal, N.; Bruder, S. P.; Mbalaviele, G.; Marshak, D. R.; Pittenger, M. F. Adult Human Mesenchymal Stem Cell Differentiation to the Osteogenic or Adipogenic Lineage Is Regulated by Mitogen-activated Protein Kinase. J. Biol. Chem. 2000, 275 (13), 9645-9652.

(63) Li, C.-S.; Zheng, Z.; Su, X.-X.; Wang, F.; Ling, M.; Zou, M.; Zhou, H. Activation of the Extracellular Signal-Regulated Kinase Signaling Is Critical for Human Umbilical Cord Mesenchymal Stem Cell Osteogenic Differentiation. BioMed Res. Int. 2016, 2016, 1-10.

(64) Hanein, D.; Horwitz, A. R. The structure of cell-matrix adhesions: the new frontier. Curr. Opin. Cell Biol. 2012, 24 (1), 134140.

(65) Chen, W.; Shao, Y.; Li, X.; Zhao, G.; Fu, J. Nanotopographical surfaces for stem cell fate control: Engineering mechanobiology from the bottom. Nano Today 2014, 9 (6), 759-784.

(66) Mammadov, R.; Mammadov, B.; Toksoz, S.; Aydin, B.; Yagci, R.; Tekinay, A. B.; Guler, M. O. Heparin Mimetic Peptide Nanofibers Promote Angiogenesis. Biomacromolecules 2011, 12 (10), 3508-3519. (67) Mammadov, R.; Mammadov, B.; Guler, M. O.; Tekinay, A. B. Growth Factor Binding on Heparin Mimetic Peptide Nanofibers. Biomacromolecules 2012, 13 (10), 3311-3319.

(68) Lim, J. Y.; Donahue, H. J. Cell sensing and response to microand nanostructured surfaces produced by chemical and topographic patterning. Tissue Eng. 2007, 13 (8), 1879-1891. 